\title{
ORIGINAL
}

\author{
Gianluca Rizzi@ • Hassam Khan • Ionel-Dumitrel Ghiba •
}

Angela Madeo - Patrizio Neff

\section{Analytical solution of the uniaxial extension problem for the relaxed micromorphic continuum and other generalized continua (including full derivations)}

Received: 19 July 2021 / Accepted: 17 October 2021 / Published online: 17 November 2021

(C) The Author(s) 2021

\begin{abstract}
We derive analytical solutions for the uniaxial extension problem for the relaxed micromorphic continuum and other generalized continua. These solutions may help in the identification of material parameters of generalized continua which are able to disclose size effects.

Keywords Generalized continua - Uniaxial extension - Uniaxial extension stiffness - Characteristic length . Size effect · Micromorphic continuum - Cosserat continuum - Couple stress model · Gradient elasticity · Micropolar · Relaxed micromorphic model - Micro-stretch model · Micro-strain model · Micro-void model . Bounded stiffness
\end{abstract}

\section{Introduction}

In this paper we continue our investigation of analytical solutions for the isotropic relaxed micromorphic model (and other isotropic generalized continuum models). It follows our recent exposition of analytical solutions for the simple shear [28], bending [25], and torsion problem [13,27]. Here, we consider the uniaxial extension problem, which, in classical isotropic linear elasticity, allows to determine the size-independent longitudinal modulus $M_{\text {macro }}=\lambda_{\text {macro }}+2 \mu_{\text {macro }}$.

Here, we show the genealogy tree of the generalized continuum models:

\author{
G. Rizzi $(\varangle)$ \\ Faculty of Architecture and Civil Engineering, TU Dortmund, August-Schmidt-Str. 8, 44227 Dortmund, Germany \\ E-mail: gianluca.rizzi@tu-dortmund.de \\ H. Khan \\ Fakultät für Mathematik, Universität Duisburg-Essen, Thea-Leymann-Straße 9, 45127 Essen, Germany \\ I. D. Ghiba \\ Department of Mathematics, Alexandru Ioan Cuza University of Iaşi, Blvd. Carol I, No. 11, 700506 Iaşi, Romania
}

I. D. Ghiba

Octav Mayer Institute of Mathematics of the Romanian Academy, Iaşi Branch, 700505 Iaşi, Romania

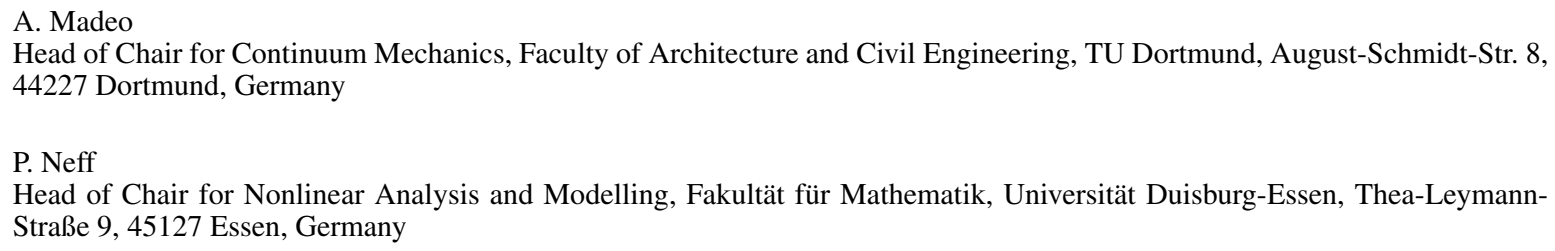



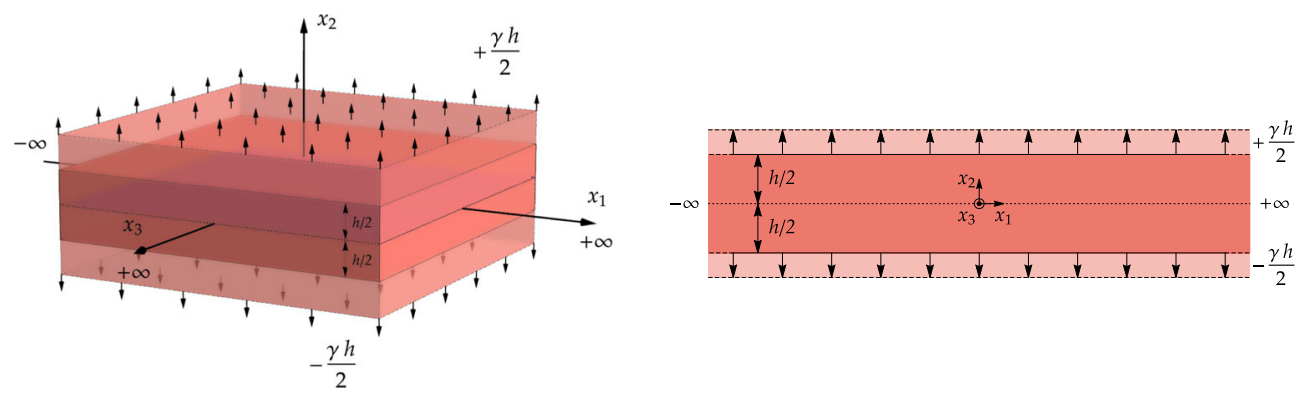

Fig. 1 Sketch of an infinite stripe of thickness $h$ subjected to uniaxial extension boundary conditions

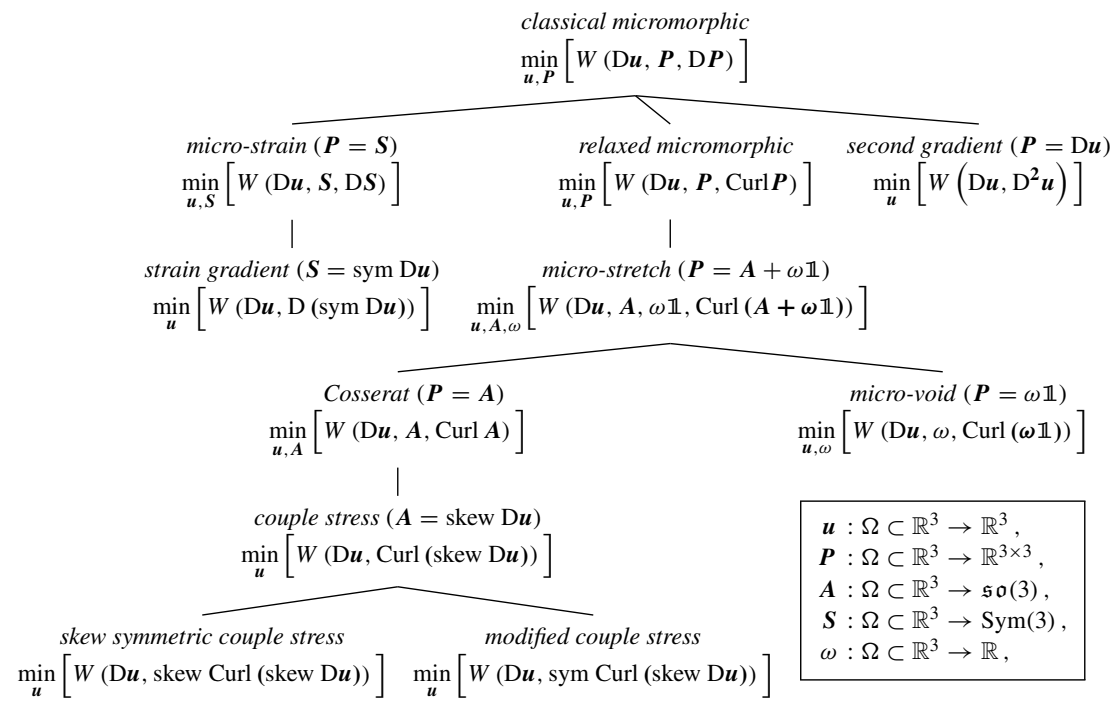

The strain gradient theory and second gradient theory are equivalent [1,17] and contain additionally the couple stress theory as a special case. Using the Curl as primary differential operator for the curvature terms allows a neat unification of concepts.

For some of the traditional models, uniaxial extension gives still rise to size effects in the sense that thinner samples are comparatively stiffer which can also be found experimentally [34-36]. In that case, the inhomogeneous response is triggered by the boundary conditions for the additional kinematic fields which are applied at the upper and lower surface. We refer the reader to the introduction of $[25,27,28,32]$ concerning the relevance of the scientific question as well as its importance for the determination of material parameters for generalized continua [33]. Indeed, the obtained analytical formulas can be used to determine size-dependent and size-independent material parameters. The notation follows that of [25,27,28]. We recapitulate shortly.

The paper is now structured as follows. We start with a recapitulation of the uniaxial extension problem in the classical linear elasticity. The solution is homogeneous and uniquely determines the longitudinal modulus $M_{\text {macro }}=\lambda_{\text {macro }}+2 \mu_{\text {macro }}$. Then, we consider the isotropic relaxed micromorphic continuum. The boundary conditions for the additional nonsymmetric micro-distortion field $\boldsymbol{P}$ derive from the so-called consistent coupling conditions

$$
\mathrm{D} u(x) \times v=P(x) \times v, \quad x \in \Gamma,
$$

where $\boldsymbol{v}$ is the normal unit vector to the upper and lower surface. It turns out that for zero Poisson modulus on the micro- and meso-scale, $v_{\text {micro }}=v_{\mathrm{e}}=0$, respectively, the solution remains homogeneous and no size effects are observed. In the case with arbitrary $v_{\text {micro }}, v_{\mathrm{e}} \in[-1,1 / 2]$ the solution will be inhomogeneous and size effects appear. The limiting stiffness as the ratio between the thickness and the characteristic length tends to zero $\left(h / L_{\mathrm{c}} \rightarrow 0\right)$ is given by $\bar{M}=\frac{M_{\mathrm{e}} M_{\text {micro }}}{M_{\mathrm{e}}+M_{\text {micro }}}$ which is both smaller than $M_{\text {micro }}=\lambda_{\text {micro }}+2 \mu_{\text {micro }}$ and $M_{\mathrm{e}}$ as well greater than $M_{\text {macro }}=\lambda_{\text {macro }}+2 \mu_{\text {macro }}$. 


\subsection{Notation}

We define the scalar product $\langle\boldsymbol{a}, \boldsymbol{b}\rangle:=\sum_{i=1}^{n} a_{i} b_{i} \in \mathbb{R}$ for vectors $a, b \in \mathbb{R}^{n}$, the dyadic product $\boldsymbol{a} \otimes \boldsymbol{b}:=\left(a_{i} b_{j}\right)_{i, j=1, \ldots, n} \in \mathbb{R}^{n \times n}$ and the Euclidean norm $\|\boldsymbol{a}\|^{2}:=\langle\boldsymbol{a}, \boldsymbol{a}\rangle$. We define the scalar product $\langle\boldsymbol{P}, \boldsymbol{Q}\rangle:=\sum_{i, j=1}^{n} P_{i j} Q_{i j} \in \mathbb{R}$ and the Frobenius-norm $\|\boldsymbol{P}\|^{2}:=\langle\boldsymbol{P}, \boldsymbol{P}\rangle$ for tensors $\boldsymbol{P}, \boldsymbol{Q} \in \mathbb{R}^{n \times n}$ in the same way. Moreover, $\boldsymbol{P}^{T}:=\left(P_{j i}\right)_{i, j=1, \ldots, n}$ denotes the transposition of the matrix $\boldsymbol{P}=\left(P_{i j}\right)_{i, j=1, \ldots, n}$, which decomposes orthogonally into the skew-symmetric part skew $\boldsymbol{P}:=\frac{1}{2}\left(\boldsymbol{P}-\boldsymbol{P}^{T}\right)$ and the symmetric part $\operatorname{sym} \boldsymbol{P}:=\frac{1}{2}\left(\boldsymbol{P}+\boldsymbol{P}^{T}\right)$. The identity matrix is denoted by $\mathbb{1}$, so that the trace of a matrix $\boldsymbol{P}$ is given by $\operatorname{tr} \boldsymbol{P}:=\langle\boldsymbol{P}, \mathbb{1}\rangle$, while the deviatoric component of a matrix is given by $\operatorname{dev} \boldsymbol{P}:=\boldsymbol{P}-\frac{\operatorname{tr}(\boldsymbol{P})}{3} \mathbb{1}$. Given this, the orthogonal decomposition possible for a matrix is $\boldsymbol{P}=\operatorname{dev} \operatorname{sym} \boldsymbol{P}+$ skew $\boldsymbol{P}+\frac{\operatorname{tr}(\boldsymbol{P})}{3} \mathbb{1}$. The Lie-algebra of skew-symmetric matrices is denoted by $\mathfrak{s o}(3):=\left\{\boldsymbol{A} \in \mathbb{R}^{3 \times 3} \mid \boldsymbol{A}^{T}=-\boldsymbol{A}\right\}$, while the vector space of symmetric matrices $\operatorname{Sym}(3):=\left\{\boldsymbol{S} \in \mathbb{R}^{3 \times 3} \mid \boldsymbol{S}^{T}=\boldsymbol{S}\right\}$. The Jacobian matrix Du and the curl for a vector field $\boldsymbol{u}$ are defined as

$$
\mathrm{D} \boldsymbol{u}=\left(\begin{array}{lll}
u_{1,1} & u_{1,2} & u_{1,3} \\
u_{2,1} & u_{2,2} & u_{2,3} \\
u_{3,1} & u_{3,2} & u_{3,3}
\end{array}\right), \quad \operatorname{curl} \boldsymbol{u}=\nabla \times \boldsymbol{u}=\left(\begin{array}{l}
u_{3,2}-u_{2,3} \\
u_{1,3}-u_{3,1} \\
u_{2,1}-u_{1,2}
\end{array}\right)
$$

where $\times$ denotes the cross-product in $\mathbb{R}^{3}$. We also introduce the Curl and the Div operators of the $3 \times 3$ matrix field $\boldsymbol{P}$ as

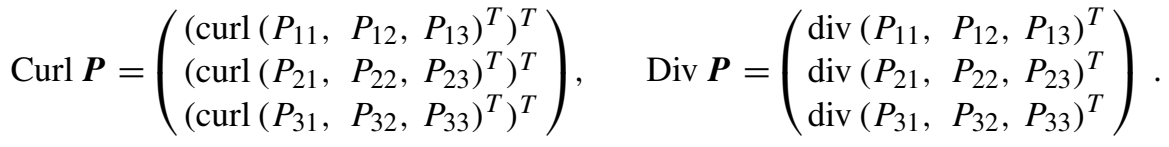

The cross-product between a second-order tensor and a vector is also needed and is defined row-wise as follows

$$
\boldsymbol{m} \times \boldsymbol{b}=\left(\begin{array}{c}
\left(b \times\left(m_{11}, m_{12}, m_{13}\right)^{T}\right)^{T} \\
\left(b \times\left(m_{21}, m_{22}, m_{23}\right)^{T}\right)^{T} \\
\left(b \times\left(m_{31}, m_{32}, m_{33}\right)^{T}\right)^{T}
\end{array}\right)=\boldsymbol{m} \cdot \boldsymbol{\epsilon} \cdot \boldsymbol{b}=m_{i k} \epsilon_{k j h} b_{h},
$$

where $\boldsymbol{m} \in \mathbb{R}^{3 \times 3}, \boldsymbol{b} \in \mathbb{R}^{3}$, and $\boldsymbol{\epsilon}$ is the Levi-Civita tensor. Using the one-to-one map axl $: \mathfrak{s o}(3) \rightarrow \mathbb{R}^{3}$ we have

$$
\boldsymbol{A} \boldsymbol{b}=\operatorname{axl}(\boldsymbol{A}) \times \boldsymbol{b} \quad \forall \boldsymbol{A} \in \mathfrak{s o}(3), \quad \boldsymbol{b} \in \mathbb{R}^{3} .
$$

The inverse of axl is denoted by Anti: $\mathbb{R}^{3} \rightarrow \mathfrak{s o}(3)$.

\section{Uniaxial extension problem for the isotropic Cauchy continuum}

The strain energy density for an isotropic Cauchy continuum is

$$
W(\mathrm{D} \boldsymbol{u})=\mu_{\text {macro }}\|\operatorname{symD} \boldsymbol{u}\|^{2}+\frac{\lambda_{\text {macro }}}{2} \operatorname{tr}^{2}(\mathrm{D} \boldsymbol{u}),
$$

while the equilibrium equations without body forces are

$$
\operatorname{Div}\left[2 \mu_{\text {macro }} \operatorname{symD} \boldsymbol{u}+\lambda_{\text {macro }} \operatorname{tr}(\mathrm{D} \boldsymbol{u}) \mathbb{1}\right]=\mathbf{0} .
$$

Since the uniaxial extensional problem is symmetric with respect to the $x_{2}$-axis, there will be no dependence of the solution on $x_{1}$ and $x_{3}$. The boundary conditions for the uniaxial extension problem are (see Fig. 1)

$$
u_{2}\left(x_{2}= \pm h / 2\right)= \pm \frac{\gamma h}{2} .
$$


The homogeneous displacement field solution $u_{2}\left(x_{2}\right)$, the gradient of the displacement $\mathrm{D} \boldsymbol{u}\left(x_{2}\right)$, and the strain energy $W(\boldsymbol{\gamma})$ for the uniaxial extension problem are

$$
\begin{aligned}
& u_{2}\left(x_{2}\right)=\boldsymbol{\gamma} x_{2}, \quad \mathrm{D} \boldsymbol{u}\left(x_{2}\right)=\left(\begin{array}{lll}
0 & 0 & 0 \\
0 & \gamma & 0 \\
0 & 0 & 0
\end{array}\right), \\
& W(\boldsymbol{\gamma})=\int_{-h / 2}^{h / 2} W(\mathrm{D} \boldsymbol{u})=\frac{1}{2}\left(\lambda_{\text {macro }}+2 \mu_{\text {macro }}\right) h \boldsymbol{\gamma}^{2}=\frac{1}{2} M_{\text {macro }} h \boldsymbol{\gamma}^{2},
\end{aligned}
$$

where

$$
M_{\text {macro }}=\lambda_{\text {macro }}+2 \mu_{\text {macro }}
$$

is the extensional stiffness (or pressure-wave modulus, longitudinal modulus).

Here and in the remainder of this work, the elastic coefficients $\mu_{i}, \lambda_{i}$ are expressed in [MPa], the coefficients $a_{i}$ and the intensity of the displacement $\gamma$ are dimensionless, the characteristic lengths $L_{\mathrm{c}}$ and the height $h$ are expressed in meter $[\mathrm{m}]$.

\section{Uniaxial extension problem for the isotropic relaxed micromorphic model}

The general expression of the strain energy for the isotropic relaxed micromorphic continuum is

$$
\begin{aligned}
W(\mathrm{D} \boldsymbol{u}, \boldsymbol{P}, \operatorname{Curl} \boldsymbol{P})= & \mu_{\mathrm{e}}\|\operatorname{sym}(\mathrm{D} \boldsymbol{u}-\boldsymbol{P})\|^{2}+\frac{\lambda_{\mathrm{e}}}{2} \operatorname{tr}^{2}(\mathrm{D} \boldsymbol{u}-\boldsymbol{P})+\mu_{\mathrm{c}}\|\operatorname{skew}(\mathrm{D} \boldsymbol{u}-\boldsymbol{P})\|^{2} \\
& +\mu_{\text {micro }}\|\operatorname{sym} \boldsymbol{P}\|^{2}+\frac{\lambda_{\text {micro }}}{2} \operatorname{tr}^{2}(\boldsymbol{P}) \\
& +\frac{\mu L_{\mathrm{c}}^{2}}{2}\left(a_{1}\|\operatorname{dev} \operatorname{sym} \operatorname{Curl} \boldsymbol{P}\|^{2}+a_{2} \| \text { skew Curl } \boldsymbol{P} \|^{2}+\frac{a_{3}}{3} \operatorname{tr}^{2}(\operatorname{Curl} \boldsymbol{P})\right),
\end{aligned}
$$

and the strictly positive definiteness conditions are ${ }^{1}$

$$
\begin{array}{ll}
\mu_{\mathrm{e}}>0, & \kappa_{\mathrm{e}}=\lambda_{\mathrm{e}}+2 / 3 \mu_{\mathrm{e}}>0, \quad \mu_{\text {micro }}>0, \quad \kappa_{\text {micro }}=\lambda_{\text {micro }}+2 / 3 \mu_{\text {micro }}>0, \\
\mu_{\mathrm{c}}>0, & \mu>0, \quad L_{\mathrm{c}}>0, \quad\left(a_{1}, a_{2}, a_{3}\right)>0 .
\end{array}
$$

where we have the parameters related to the meso-scale, the parameters related to the micro-scale, the Cosserat couple modulus, the proportionality stiffness parameter, the characteristic length and the three dimensionless general isotropic curvature parameters, respectively. This energy expression represents the most general isotropic form possible for the relaxed micromorphic model. In the absence of body forces, the equilibrium equations are then

$$
\begin{aligned}
& \operatorname{Div} \overbrace{\left[2 \mu_{\mathrm{e}} \operatorname{sym}(\operatorname{D} \boldsymbol{u}-\boldsymbol{P})+\lambda_{\mathrm{e}} \operatorname{tr}(\mathrm{D} \boldsymbol{u}-\boldsymbol{P}) \mathbb{1}+2 \mu_{\mathrm{c}} \operatorname{skew}(\mathrm{D} \boldsymbol{u}-\boldsymbol{P})\right]}^{\tilde{\sigma}:=}=\mathbf{0}, \\
& \tilde{\sigma}-2 \mu_{\text {micro }} \operatorname{sym} \boldsymbol{P}-\lambda_{\text {micro } \operatorname{tr}(\boldsymbol{P}) \mathbb{1}} \\
& -\mu L_{\mathrm{c}}^{2} \operatorname{Curl}\left(a_{1} \operatorname{dev} \operatorname{sym} \operatorname{Curl} \boldsymbol{P}+a_{2} \text { skew } \operatorname{Curl} \boldsymbol{P}+a_{3} \operatorname{tr}(\operatorname{Curl} \boldsymbol{P})\right)=\mathbf{0} .
\end{aligned}
$$

The ansatz for the micro-distortion $\boldsymbol{P}\left(x_{2}\right)$, the displacement $\boldsymbol{u}\left(x_{2}\right)$, and consequently the gradient of the displacement $\mathrm{D} \boldsymbol{u}\left(x_{2}\right)$ is

$$
\begin{aligned}
\boldsymbol{u}\left(x_{2}\right) & =\left(\begin{array}{c}
0 \\
u_{2}\left(x_{2}\right) \\
0
\end{array}\right), \\
\mathrm{D} \boldsymbol{u}\left(x_{2}\right) & =\left(\begin{array}{cccc}
0 & 0 & 0 \\
0 & u_{2,2}\left(x_{2}\right) & 0 \\
0 & 0 & 0
\end{array}\right) .
\end{aligned}
$$

\footnotetext{
1 Note that the model has a unique solution including the case of a Cosserat couple modulus $\mu_{\mathrm{c}}=0$.
} 
It is important to underline that, given subsequent ansatz (14), it holds that $\operatorname{tr}(\mathrm{Curl} \boldsymbol{P})=0$. This reduces immediately the number of curvature parameters appearing in the uniaxial extension solution.

The boundary conditions for the uniaxial extension are

$$
u_{2}\left(x_{2}= \pm h / 2\right)= \pm \frac{\gamma h}{2}, \quad P_{11}\left(x_{2}= \pm h / 2\right)=0, \quad P_{33}\left(x_{2}= \pm h / 2\right)=0 .
$$

Here, the constraint on the components of $\boldsymbol{P}$ is given by the consistent coupling boundary condition

$$
\boldsymbol{P} \times \boldsymbol{v}=\mathrm{D} \boldsymbol{u} \times \boldsymbol{v}, \quad\left(\begin{array}{ccc}
0 & 0 & 0 \\
0 & 0 & 0 \\
0 & 0 & 0
\end{array}\right)=\left(\begin{array}{ccc}
0 & 0 & P_{11} \\
0 & 0 & 0 \\
-P_{33} & 0 & 0
\end{array}\right),
$$

where $\boldsymbol{v}$ is the normal unit vector to the upper and lower surface.

After substituting ansatz (14) into equilibrium equation (13) we obtain the following four differential equations

$$
\begin{aligned}
M_{\mathrm{e}}\left(u_{2}^{\prime \prime}\left(x_{2}\right)-P_{22}^{\prime}\left(x_{2}\right)\right)-\lambda_{\mathrm{e}}\left(P_{11}^{\prime}\left(x_{2}\right)+P_{33}^{\prime}\left(x_{2}\right)\right) & =0, \\
\frac{1}{2} \mu L_{\mathrm{c}}^{2}\left(\left(a_{1}+a_{2}\right) P_{11}^{\prime \prime}\left(x_{2}\right)+\left(a_{2}-a_{1}\right) P_{33}^{\prime \prime}\left(x_{2}\right)\right) & \\
-\left(M_{\mathrm{e}}+M_{\text {micro }}\right) P_{11}\left(x_{2}\right)-\left(\lambda_{\mathrm{e}}+\lambda_{\text {micro }}\right)\left(P_{22}\left(x_{2}\right)+P_{33}\left(x_{2}\right)\right)+\lambda_{\mathrm{e}} u_{2}^{\prime}\left(x_{2}\right) & =0, \\
-\left(M_{\mathrm{e}}+M_{\text {micro }}\right) P_{22}\left(x_{2}\right)+M_{\mathrm{e}} u_{2}^{\prime}\left(x_{2}\right)-\left(\lambda_{\mathrm{e}}+\lambda_{\text {micro }}\right)\left(P_{11}\left(x_{2}\right)+P_{33}\left(x_{2}\right)\right) & =0, \\
\frac{1}{2} \mu L_{\mathrm{c}}^{2}\left(\left(a_{2}-a_{1}\right) P_{11}^{\prime \prime}\left(x_{2}\right)+\left(a_{1}+a_{2}\right) P_{33}^{\prime \prime}\left(x_{2}\right)\right) & \\
-\left(M_{\mathrm{e}}+M_{\text {micro }}\right) P_{33}\left(x_{2}\right)-\left(\lambda_{\mathrm{e}}+\lambda_{\text {micro }}\right)\left(P_{11}\left(x_{2}\right)+P_{22}\left(x_{2}\right)\right)+\lambda_{\mathrm{e}} u_{2}^{\prime}\left(x_{2}\right) & =0,
\end{aligned}
$$

where $M_{\mathrm{e}}=\lambda_{\mathrm{e}}+2 \mu_{\mathrm{e}}$ and $M_{\text {micro }}=\lambda_{\text {micro }}+2 \mu_{\text {micro }}$. Being careful of substituting the system of differential equation with one in which Eq. (17) ${ }_{2}$ and Eq. (17) 4 are replaced with their sum and their difference, respectively, we have

$$
\begin{aligned}
M_{\mathrm{e}}\left(u_{2}^{\prime \prime}\left(x_{2}\right)-P_{22}^{\prime}\left(x_{2}\right)\right)-\lambda_{\mathrm{e}} f_{p}^{\prime}\left(x_{2}\right) & =0, \\
a_{2} \mu L_{\mathrm{c}}^{2} f_{p}^{\prime \prime}\left(x_{2}\right)-\left(M_{\mathrm{e}}+\lambda_{\mathrm{e}}+M_{\text {micro }}+\lambda_{\text {micro }}\right) f_{p}\left(x_{2}\right)-2\left(\lambda_{\mathrm{e}}+\lambda_{\text {micro }}\right) P_{22}\left(x_{2}\right)+2 \lambda_{\mathrm{e}} u_{2}^{\prime}\left(x_{2}\right) & =0, \\
-\left(M_{\mathrm{e}}+M_{\text {micro }}\right) P_{22}\left(x_{2}\right)+M_{\mathrm{e}} u_{2}^{\prime}\left(x_{2}\right)-\left(\lambda_{\mathrm{e}}+\lambda_{\text {micro }}\right) f_{p}\left(x_{2}\right) & =0, \\
a_{1} \mu L_{\mathrm{c}}^{2} f_{m}^{\prime \prime}\left(x_{2}\right)-\left(M_{\text {micro }}+M_{\mathrm{e}}-\lambda_{\mathrm{e}}-\lambda_{\text {micro }}\right) f_{m}\left(x_{2}\right) & =0,
\end{aligned}
$$

where $f_{p}\left(x_{2}\right):=P_{11}\left(x_{2}\right)+P_{33}\left(x_{2}\right)$ and $f_{m}\left(x_{2}\right):=P_{11}\left(x_{2}\right)-P_{33}\left(x_{2}\right)$. It is highlighted that Eq. (18) $)_{4}$ is a homogeneous second-order differential equation depending only on $f_{m}\left(x_{2}\right)$ with homogeneous boundary conditions Eq. (15).

The fact that Eq. (18) 4 is an independent equation has its meaning in the symmetry constraint of the uniaxial extensional problem in the direction along the $x_{2}$ - and $x_{3}$-axis, which requires that $P_{11}\left(x_{2}\right)=P_{33}\left(x_{2}\right)$. From Eq. (18) it is possible to obtain the following relation between $P_{22}\left(x_{2}\right)$ and $u_{2}\left(x_{2}\right)$

$$
P_{22}\left(x_{2}\right)=\frac{M_{\mathrm{e}} u_{2}^{\prime}\left(x_{2}\right)-\left(\lambda_{\mathrm{e}}+\lambda_{\text {micro }}\right) f_{p}\left(x_{2}\right)}{M_{\mathrm{e}}+M_{\text {micro }}},
$$

which, after substituting it back into Eq. (18), allows us to obtain the following system of three second-order differential equations in $u_{2}\left(x_{2}\right), P_{22}\left(x_{2}\right)$, and $f_{p}\left(x_{2}\right)$

$$
\begin{aligned}
z_{1} f_{p}^{\prime}\left(x_{2}\right)+z_{2} u_{2}^{\prime \prime}\left(x_{2}\right) & =0, \\
a_{2} \mu L_{\mathrm{c}}^{2} f_{p}^{\prime \prime}\left(x_{2}\right)-z_{3} f_{p}\left(x_{2}\right)-2 z_{1} u_{2}^{\prime}\left(x_{2}\right) & =0, \\
a_{1} \mu L_{\mathrm{c}}^{2} f_{m}^{\prime \prime}\left(x_{2}\right)-\left(M_{\mathrm{e}}+M_{\text {micro }}-\lambda_{\mathrm{e}}-\lambda_{\text {micro }}\right) f_{m}\left(x_{2}\right) & =0,
\end{aligned}
$$


where

$$
\begin{aligned}
& z_{1}:=\frac{M_{\mathrm{e}} \lambda_{\text {micro }}-\lambda_{\mathrm{e}} M_{\text {micro }}}{M_{\mathrm{e}}+M_{\text {micro }}}, \quad z_{2}:=\frac{M_{\mathrm{e}} M_{\text {micro }}}{M_{\mathrm{e}}+M_{\text {micro }}}, \\
& z_{3}:=\frac{\left(M_{\mathrm{e}}-\lambda_{\mathrm{e}}+M_{\text {micro }}-\lambda_{\text {micro }}\right)\left(M_{\mathrm{e}}+2 \lambda_{\mathrm{e}}+M_{\text {micro }}+2 \lambda_{\text {micro }}\right)}{M_{\mathrm{e}}+M_{\text {micro }}} .
\end{aligned}
$$

It is highlighted that due to positive definiteness conditions (12), $\left(z_{2}, z_{3}\right)>0$ and $z_{1}=0$ if and only if $\lambda_{\text {micro }}=\lambda_{\mathrm{e}}=0$ (zero Poisson's ratio case which is studied in Sect. 3.1) and $\frac{M_{\text {micro }}}{M_{\mathrm{e}}}=\frac{\lambda_{\text {micro }}}{\lambda_{\mathrm{e}}}$. If $z_{1}$ is zero, Eq. (20) uncouples completely into three independent differential equations in $u_{2}, f_{\mathrm{p}}$, and $f_{\mathrm{m}}$, respectively.

After applying boundary conditions Eq. (15), the solution in terms of $u_{2}\left(x_{2}\right), P_{11}\left(x_{2}\right), P_{22}\left(x_{2}\right)$, and $P_{33}\left(x_{2}\right)$ of system Eq. (20) is ${ }^{2}$

$$
\begin{aligned}
u_{2}\left(x_{2}\right) & =\frac{\frac{2 x_{2}}{h}-\frac{4 z_{1}^{2}}{f_{1} z_{2} z_{3}} \operatorname{sech}\left(\frac{f_{1} h}{2 L_{\mathrm{c}}}\right) \sinh \left(\frac{f_{1} x_{2}}{L_{\mathrm{c}}}\right) \frac{L_{\mathrm{c}}}{h}}{1-\frac{4 z_{1}^{2}}{f_{1} z_{2} z_{3}} \tanh \left(\frac{f_{1} h}{2 L_{\mathrm{c}}}\right) \frac{L_{\mathrm{c}}}{h}}, \\
P_{22}\left(x_{2}\right) & =\frac{M_{\mathrm{e}}+2 \frac{z_{1}}{z_{3}}\left(\lambda_{\mathrm{e}}+\lambda_{\text {micro }}\right)-\frac{z_{1}}{z_{3}}\left(M_{\mathrm{e}} \frac{2 z_{1}}{z_{2}}+2\left(\lambda_{\mathrm{e}}+\lambda_{\text {micro }}\right) \cosh \left(\frac{f_{1} h}{L_{\mathrm{c}}}\right) \operatorname{sech}\left(\frac{f_{1} h}{2 L_{\mathrm{c}}}\right)\right)}{\left(M_{\mathrm{e}}+M_{\text {micro }}\right)\left(1-\frac{4 z_{1}^{2}}{f_{1} z_{2} z_{3}} \tanh \left(\frac{f_{1} h}{2 L_{\mathrm{c}}}\right) \frac{L_{\mathrm{c}}}{h}\right)} \boldsymbol{\gamma}, \\
P_{11}\left(x_{2}\right) & =P_{33}\left(x_{2}\right)=\frac{\frac{z_{1}}{z_{3}}\left(\operatorname{sech}\left(\frac{f_{1} h}{2 L_{\mathrm{c}}}\right) \cosh \left(\frac{f_{1} x_{2}}{L_{\mathrm{c}}}\right)-1\right)}{1-\frac{4 z_{1}^{2}}{f_{1} z_{2} z_{3}} \tanh \left(\frac{f_{1} h}{2 L_{\mathrm{c}}}\right) \frac{L_{\mathrm{c}}}{h}} \boldsymbol{\gamma}, \quad f_{1}:=\sqrt{\frac{z_{2} z_{3}-2 z_{1}^{2}}{\mu a_{2} z_{2}}} .
\end{aligned}
$$

In the above expressions all the quantities are real and well defined due to positive definiteness conditions Eq. (12). Indeed, since the coefficients $z_{1}, z_{2}$, and $z_{3}$ may be rewritten in terms of the meso- and micro-bulk and shear modulus as

$$
\begin{aligned}
& z_{1}:=\frac{6 \kappa_{\text {micro }} \mu_{\mathrm{e}}-6 \kappa_{\mathrm{e}} \mu_{\text {micro }}}{3 \kappa_{\mathrm{e}}+3 \kappa_{\text {micro }}+4\left(\mu_{\mathrm{e}}+\mu_{\text {micro }}\right)}, \quad z_{2}:=\frac{\left(3 \kappa_{\mathrm{e}}+4 \mu_{\mathrm{e}}\right)\left(3 \kappa_{\text {micro }}+4 \mu_{\text {micro }}\right)}{9 \kappa_{\mathrm{e}}+9 \kappa_{\text {micro }}+12\left(\mu_{\mathrm{e}}+\mu_{\text {micro }}\right)}, \\
& z_{3}:=\frac{18\left(\kappa_{\mathrm{e}}+\kappa_{\text {micro }}\right)\left(\mu_{\mathrm{e}}+\mu_{\text {micro }}\right)}{3 \kappa_{\mathrm{e}}+3 \kappa_{\text {micro }}+4\left(\mu_{\mathrm{e}}+\mu_{\text {micro }}\right)},
\end{aligned}
$$

we can write the expression of $f_{1}$ as follows

$$
f_{1}:=\sqrt{\frac{6 \kappa_{\mathrm{e}} \kappa_{\text {micro }}\left(\mu_{\mathrm{e}}+\mu_{\text {micro }}\right)+8 \mu_{\mathrm{e}} \mu_{\text {micro }}\left(\kappa_{\mathrm{e}}+\kappa_{\text {micro }}\right)}{\mu a_{2}\left(\kappa_{\mathrm{e}}+\frac{4}{3} \mu_{\mathrm{e}}\right)\left(\kappa_{\text {micro }}+\frac{4}{3} \mu_{\text {micro }}\right)}},
$$

showing that the positive definiteness of energy (11) implies that $f_{1}$ is a strictly positive real number. Moreover, the function $g:(0, \infty) \rightarrow \mathbb{R}, \quad g(x):=1-\frac{4 z_{1}^{2}}{z_{2} z_{3}} \frac{1}{x} \tanh \frac{x}{2}$ has the asymptotic behavior

$$
\lim _{x \rightarrow 0} g(x)=1-\frac{2 z_{1}^{2}}{z_{2} z_{3}}=f_{1}^{2}>0, \quad \lim _{x \rightarrow \infty} g(x)=1
$$

and it is monotone increasing since its first derivative is given by

$$
g^{\prime}(x)=\frac{4 z_{1}^{2}}{z_{2} z_{3}} \frac{\sinh x-x}{x^{2}(\cosh x+1)}
$$

which it is positive for all $x \in(0, \infty)$. Hence, it follows that due to the positive definiteness of the elastic energy

$$
g(x)>0 \quad \forall x>0
$$

\footnotetext{
${ }^{2} \operatorname{sech}(x)=1 / \cosh (x)$.
} 

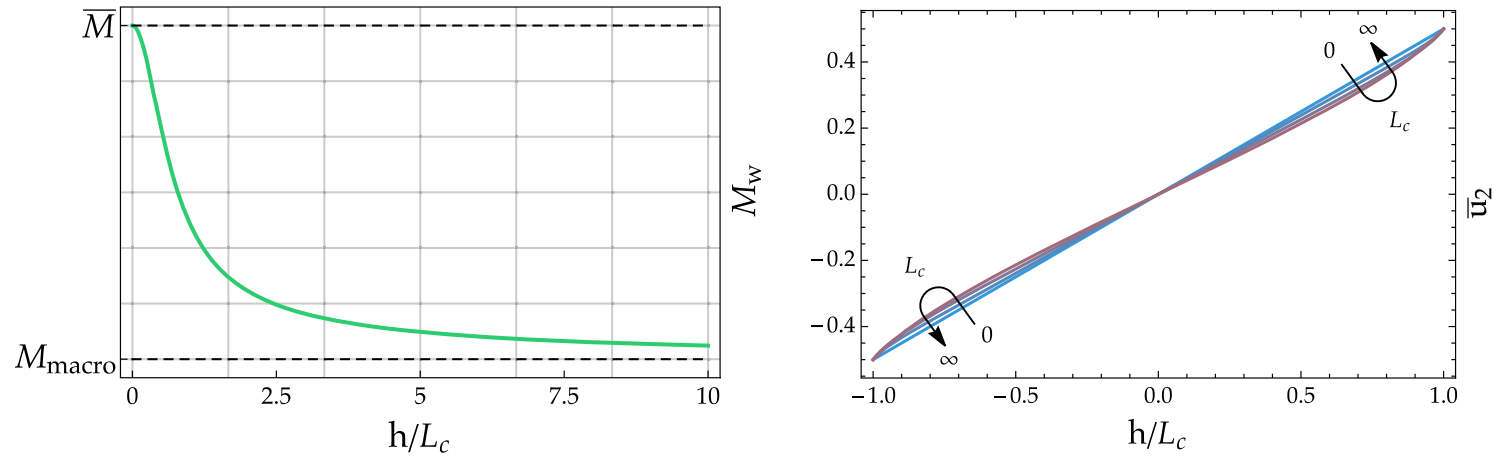

Fig. 2 Relaxed micromorphic model. (left) Extensional stiffness $M_{\mathrm{w}}$ while varying $L_{\mathrm{c}}$. The stiffness is bounded as $L_{\mathrm{c}} \rightarrow \infty$ $(h \rightarrow 0)$. The values of the parameters used are: $\mu=1, \lambda_{\mathrm{e}}=1, M_{\mathrm{e}}=2, \lambda_{\text {micro }}=3, M_{\text {micro }}=4, a_{2}=1 / 5 ;($ right $)$ Displacement profile across the thickness of the dimensionless $\bar{u}_{2}=u_{2} /(\gamma h)$ for different values of $L_{\mathrm{c}}=\{0,0.014,0.0 \overline{3}, 0.1\}$. The values of the other parameters used in order to maximize the nonhomogeneous behavior are $\mu=1, \lambda_{\mathrm{e}}=1, M_{\mathrm{e}}=1, \lambda_{\text {micro }}=0.001$, $M_{\text {micro }}=0.056, a_{2}=0 . \overline{3}$

which implies that

$$
1-\frac{4 z_{1}^{2}}{f_{1} z_{2} z_{3}} \tanh \left(\frac{f_{1} h}{2 L_{\mathrm{c}}}\right) \frac{L_{\mathrm{c}}}{h}>0 \quad \forall L_{\mathrm{c}}>0
$$

which completes our proof that all the quantities from (22) are real and well-defined.

The strain energy associated with this solution is

$$
\begin{aligned}
W(\boldsymbol{\gamma})= & \int_{-h / 2}^{h / 2} W(\mathrm{D} \boldsymbol{u}, \boldsymbol{P}, \operatorname{Curl} \boldsymbol{P}) \\
= & \frac{1}{2}\left[\frac{\mu a_{2}\left(\frac{f_{1} z_{1}}{z_{3}}\right)^{2}\left(\frac{1}{f_{1}} \sinh \left(\frac{f_{1} h}{L_{\mathrm{c}}}\right) \frac{L_{\mathrm{c}}}{h}-1\right)}{\left(1-\frac{4 z_{1}^{2}}{f_{1} z_{23}} \tanh \left(\frac{f_{1} h}{2 L_{\mathrm{c}}}\right) \frac{L_{\mathrm{c}}}{h}\right)^{2} \cosh ^{2}\left(\frac{f_{1} h}{2 L_{\mathrm{c}}}\right)}+\frac{\cosh ^{2}\left(\frac{f_{1} h}{2 L_{\mathrm{c}}}\right)-\frac{z_{1}^{2}}{z_{2} z_{3}}\left(\frac{3}{f_{1}} \sinh \left(\frac{f_{1} h}{L_{\mathrm{c}}}\right) \frac{L_{\mathrm{c}}}{h}-1\right)}{\left(1-\frac{4 z_{1}^{2}}{f_{1} z_{2} z_{3}} \tanh \left(\frac{f_{1} h}{2 L_{\mathrm{c}}}\right) \frac{L_{\mathrm{c}}}{h}\right)^{2} \cosh ^{2}\left(\frac{f_{1} h}{2 L_{\mathrm{c}}}\right)}\right. \\
& \left.\times \frac{z_{2}}{z_{3}}\left(M_{\mathrm{e}}+M_{\text {micro }}+\lambda_{\mathrm{e}}+\lambda_{\text {micro }}-\frac{2 \lambda_{\mathrm{e}}^{2}}{M_{\mathrm{e}}}-\frac{2 \lambda_{\text {micro }}^{2}}{M_{\text {micro }}}\right)\right] h \boldsymbol{\gamma}^{2}=\frac{1}{2} M_{\mathrm{w}} h \boldsymbol{\gamma}^{2} .
\end{aligned}
$$

The plot of the extensional stiffness $M_{\mathrm{w}}$ while varying $L_{\mathrm{c}}$ is shown in Fig. 2.

The values of $M_{\text {macro }}$ and $M_{\text {micro }}$ are

$$
\begin{aligned}
M_{\text {macro }}=\lim _{L_{\mathrm{c}} \rightarrow 0} M_{\mathrm{w}} & =\frac{M_{\mathrm{e}}^{2} M_{\text {micro }}+M_{\mathrm{e}}\left(-2 \lambda_{\text {micro }}^{2}+M_{\text {micro }}^{2}+M_{\text {micro }}\left(\lambda_{\mathrm{e}}+\lambda_{\text {micro }}\right)\right)-2 \lambda_{\mathrm{e}}^{2} M_{\text {micro }}}{\left(M_{\mathrm{e}}-\lambda_{\mathrm{e}}-\lambda_{\text {micro }}+M_{\text {micro }}\right)\left(M_{\mathrm{e}}+2\left(\lambda_{\mathrm{e}}+\lambda_{\text {micro }}\right)+M_{\text {micro }}\right)} \\
& =\frac{\kappa_{\mathrm{e}} \kappa_{\text {micro }}}{\kappa_{\mathrm{e}}+\kappa_{\text {micro }}}+\frac{4}{3} \frac{\mu_{\mathrm{e}} \mu_{\text {micro }}}{\mu_{\mathrm{e}}+\mu_{\text {micro }}}=\kappa_{\text {macro }}+\frac{4}{3} \mu_{\text {macro }}=M_{\text {macro }}, \\
\bar{M}=\lim _{L_{\mathrm{c}} \rightarrow \infty} M_{\mathrm{w}} & =\frac{M_{\mathrm{e}} M_{\text {micro }}}{M_{\mathrm{e}}+M_{\text {micro }}}<\left\{\begin{array}{l}
M_{\text {micro }} \\
M_{\mathrm{e}}
\end{array}\right.
\end{aligned}
$$

where $M_{i}=\kappa_{i}+\frac{4}{3} \mu_{i}$ and $\lambda_{i}=\kappa_{i}-\frac{2}{3} \mu_{i}$ with $i=$ \{macro, micro, e $\}$.

It is highlighted that the structure $\frac{(\bullet)_{\mathrm{e}}(\bullet)_{\text {micro }}}{(\bullet)_{\mathrm{e}}+\left(\bullet \bullet_{\text {micro }}\right.}$ is applicable to evaluate the macro coefficients only for the shear and bulk modulus because of the orthogonal energy decomposition "sym dev/tr" of which they are related, and especially here it would be a mistake to use this structure for the coefficient $M_{\text {macro }}$ since it will give the value at the micro-scale. For more details about $\lim _{L_{\mathrm{c}} \rightarrow \infty} M_{\mathrm{w}}$ see Appendix A.

\footnotetext{
3 For the sake of completeness are reported here also the relations between the Young's modulus $E_{i}$ and the Poisson's ratio $v_{i}$ in terms of $\kappa_{i}$ and $\mu_{i}: E_{i}=\frac{9 \kappa_{i} \mu_{i}}{3 \kappa_{i}+\mu_{i}}$ and $v_{i}=\frac{3 \kappa_{i}-2 \mu_{i}}{2\left(3 \kappa_{i}+\mu_{i}\right)}$ with $i=\{$ macro, micro, e $\}$.
} 
3.1 Uniaxial extension problem for the isotropic relaxed micromorphic model with $v_{e}=v_{\text {micro }}=0$

A vanishing Poisson's ratio at the meso- and micro-scale $\left(v_{e}=v_{\text {micro }}=0\right)$ corresponds to a vanishing first Lamé parameter $\left(\lambda_{\mathrm{e}}=\lambda_{\text {micro }}=0\right)$. It is easy to see from Eqs. (21) and (22) that these conditions correspond to

$$
\lambda_{\mathrm{e}}=\lambda_{\text {micro }}=0 \Longleftrightarrow\left\{\begin{array}{l}
z_{1}=0, \\
z_{2}=\frac{M_{\mathrm{e}} M_{\text {micro }}}{M_{\mathrm{e}}+M_{\text {micro }}}=\frac{2 \mu_{\mathrm{e}} \mu_{\text {micro }}}{\mu_{\mathrm{e}}+\mu_{\text {micro }}}, \\
z_{3}=M_{\mathrm{e}}+M_{\text {micro }}=2\left(\mu_{\mathrm{e}}+\mu_{\text {micro }}\right),
\end{array}\right.
$$

with $M_{i}=\lambda_{i}+2 \mu_{i}=2 \mu_{i}$ with $i=$ \{micro, e\}. Since the nonlinear terms in solution Eq. (22) vanish, we retrieve

$$
u_{2}\left(x_{2}\right)=\boldsymbol{\gamma} x_{2}, \quad P_{22}\left(x_{2}\right)=\frac{\mu_{\mathrm{e}}}{\mu_{\mathrm{e}}+\mu_{\text {micro }}} \boldsymbol{\gamma}, \quad P_{11}\left(x_{2}\right)=P_{33}\left(x_{2}\right)=0,
$$

which is a homogeneous elastic solution satisfying the equilibrium equation in the case of a constant microdistortion tensor $\overline{\boldsymbol{P}}$ (see Appendix D of [27] for further details)

$$
\overline{\boldsymbol{P}}=\frac{\mu_{\mathrm{e}}}{\mu_{\mathrm{e}}+\mu_{\text {micro }}}\left(\frac{1}{|\Omega|} \int_{\Omega} \mathrm{D} \boldsymbol{u} \mathrm{d} V\right) .
$$

The strain energy associated with this solution is

$$
W(\boldsymbol{\gamma})=\int_{-h / 2}^{h / 2} W(\mathrm{D} \boldsymbol{u})=\frac{1}{2} \frac{2 \mu_{\mathrm{e}} \mu_{\text {micro }}}{\mu_{\mathrm{e}}+\mu_{\text {micro }}} h \boldsymbol{\gamma}^{2}=\frac{1}{2} M_{\text {macro }} h \boldsymbol{\gamma}^{2},
$$

where $M_{\text {macro }}=2 \mu_{\text {macro }}+\lambda_{\text {macro }}=2 \mu_{\text {macro }}=\frac{2 \mu_{\mathrm{e}} \mu_{\text {micro }}}{\mu_{\mathrm{e}}+\mu_{\text {micro }}}$ is the macro-extensional stiffness, since $\lambda_{\text {macro }}=$ $v_{\text {macro }}=0$.

\section{Uniaxial extension problem for the isotropic micro-stretch model in dislocation format}

In the micro-stretch model in dislocation format [5,15,20,22,30], the micro-distortion tensor $\boldsymbol{P}$ is devoid from the deviatoric component dev $\operatorname{sym} \boldsymbol{P}=0 \Leftrightarrow \boldsymbol{P}=\boldsymbol{A}+\omega \mathbb{1}, \boldsymbol{A} \in \mathfrak{s o}(3), \omega \in \mathbb{R}$. The expression of the strain energy for this model in dislocation format can be written as [20]:

$$
\begin{aligned}
W & (\mathrm{D} \boldsymbol{u}, \boldsymbol{A}, \omega, \operatorname{Curl}(\boldsymbol{A}-\omega \mathbb{1})) \\
= & \mu_{\text {macro }}\|\operatorname{dev} \operatorname{sym} \mathrm{D} \boldsymbol{u}\|^{2}+\frac{\kappa_{\mathrm{e}}}{2} \operatorname{tr}^{2}(\mathrm{D} \boldsymbol{u}-\omega \mathbb{1})+\mu_{c} \| \text { skew }(\mathrm{D} \boldsymbol{u}-\boldsymbol{A}) \|^{2}+\frac{9}{2} \kappa_{\text {micro }} \omega^{2} \\
& +\frac{\mu L_{c}^{2}}{2}\left(a_{1}\|\operatorname{dev} \operatorname{sym} \operatorname{Curl} \boldsymbol{A}\|^{2}+a_{2} \| \text { skew Curl }(\boldsymbol{A}+\omega \mathbb{1}) \|^{2}+\frac{a_{3}}{3} \operatorname{tr}^{2}(\operatorname{Curl} \boldsymbol{A})\right),
\end{aligned}
$$

since $\operatorname{Curl}(\omega \mathbb{1}) \in \mathfrak{s o}(3)$. The equilibrium equations, in the absence of body forces, are then

$$
\begin{aligned}
& \operatorname{Div} \overbrace{\left[2 \mu_{\text {macro }} \operatorname{dev} \operatorname{sym} \operatorname{D} \boldsymbol{u}+\kappa_{\mathrm{e}} \operatorname{tr}(\operatorname{D} \boldsymbol{u}-\omega \mathbb{1}) \mathbb{1}+2 \mu_{c} \operatorname{skew}(\operatorname{D} \boldsymbol{u}-\boldsymbol{A})\right]}^{\tilde{\sigma}:=}=\mathbf{0}, \\
& 2 \mu_{c} \operatorname{skew}(\operatorname{D} \boldsymbol{u}-\boldsymbol{A}) \\
& \quad-\mu L_{c}^{2} \operatorname{skew} \operatorname{Curl}\left(a_{1} \operatorname{dev} \operatorname{sym} \operatorname{Curl} \boldsymbol{A}+a_{2} \operatorname{skew} \operatorname{Curl}(\boldsymbol{A}+\omega \mathbb{1})+\frac{a_{3}}{3} \operatorname{tr}(\operatorname{Curl} \boldsymbol{A}) \mathbb{1}\right)=\mathbf{0} \\
& \operatorname{tr}\left[2 \mu_{\text {macro }} \operatorname{dev} \operatorname{sym} \operatorname{D} \boldsymbol{u}\right. \\
& \left.\quad+\kappa_{\mathrm{e}} \operatorname{tr}(\operatorname{D} \boldsymbol{u}-\omega \mathbb{1}) \mathbb{1}-\kappa_{\text {micro }} \operatorname{tr}(\omega \mathbb{1}) \mathbb{1}-\mu L_{c}^{2} a_{2} \operatorname{Curl~skew~} \operatorname{Curl}(\omega \mathbb{1}+\boldsymbol{A})\right]=\mathbf{0} .
\end{aligned}
$$


According to the reference system shown in Fig. 1, the ansatz for the displacement and micro-distortion fields is

$$
\begin{aligned}
\boldsymbol{u}\left(x_{2}\right) & =\left(\begin{array}{c}
0 \\
u_{2}\left(x_{2}\right) \\
0
\end{array}\right), & \boldsymbol{A}\left(x_{2}\right) & =\left(\begin{array}{ccc}
0 & 0 & 0 \\
0 & 0 & 0 \\
0 & 0 & 0
\end{array}\right), \\
\mathrm{D} \boldsymbol{u}\left(x_{2}\right) & =\left(\begin{array}{ccc}
0 & 0 & 0 \\
0 & u_{2,2}\left(x_{2}\right) & 0 \\
0 & 0 & 0
\end{array}\right), & \omega\left(x_{2}\right) \mathbb{1} & =\left(\begin{array}{ccc}
\omega\left(x_{2}\right) & 0 & 0 \\
0 & \omega\left(x_{2}\right) & 0 \\
0 & 0 & \omega\left(x_{2}\right)
\end{array}\right) .
\end{aligned}
$$

The boundary conditions at the free surface are then

$$
u_{2}\left(x_{2}= \pm h / 2\right)= \pm \frac{\gamma h}{2}, \quad \omega\left(x_{2}= \pm h / 2\right)=0 .
$$

Since the ansatz requires $\boldsymbol{A}=0$, the micro-stretch model coincides with the micro-void model which will be presented in Sect. 6 .

\section{Uniaxial extension problem for the isotropic Cosserat continuum}

The strain energy for the isotropic Cosserat continuum in dislocation tensor format (curvature energy expressed in terms of Curl $\boldsymbol{A})$ can be written as $[3,8,13,14,18,21,25,28,29]$

$$
\begin{aligned}
W(\mathrm{D} \boldsymbol{u}, \boldsymbol{A}, \operatorname{Curl} \boldsymbol{A})= & \mu_{\text {macro }}\|\operatorname{sym} \mathrm{D} \boldsymbol{u}\|^{2}+\frac{\lambda_{\text {macro }}}{2} \operatorname{tr}^{2}(\mathrm{D} \boldsymbol{u})+\mu_{\mathrm{c}} \| \text { skew }(\mathrm{D} \boldsymbol{u}-\boldsymbol{A}) \|^{2} \\
& +\frac{\mu L_{\mathrm{c}}^{2}}{2}\left(a_{1}\|\operatorname{dev} \operatorname{sym} \operatorname{Curl} \boldsymbol{A}\|^{2}+a_{2} \| \text { skew Curl } \boldsymbol{A} \|^{2}+\frac{a_{3}}{3} \operatorname{tr}^{2}(\operatorname{Curl} \boldsymbol{A})\right),
\end{aligned}
$$

where $\boldsymbol{A} \in \mathfrak{s o}$ (3). The equilibrium equations, in the absence of body forces, are therefore the following

$$
\begin{aligned}
& \operatorname{Div} \overbrace{\left[2 \mu_{\text {macro }} \operatorname{sym} \mathrm{D} \boldsymbol{u}+\lambda_{\text {macro }} \operatorname{tr}(\mathrm{D} \boldsymbol{u}) \mathbb{1}+2 \mu_{\mathrm{c}} \operatorname{skew}(\mathrm{D} \boldsymbol{u}-\boldsymbol{A})\right]}^{\widetilde{\sigma}:=}=\mathbf{0}, \\
& 2 \mu_{\mathrm{c}} \text { skew }(\mathrm{D} \boldsymbol{u}-\boldsymbol{A})-\mu L_{\mathrm{c}}^{2} \text { skew Curl }\left(a_{1} \operatorname{dev} \operatorname{sym} \operatorname{Curl} \boldsymbol{A}+\frac{a_{3}}{3} \operatorname{tr}(\operatorname{Curl} \boldsymbol{A}) \mathbb{1}\right)=\mathbf{0} \text {. }
\end{aligned}
$$

According to the reference system shown in Fig. 1 and ansatz (14), which has to be particularized as $\boldsymbol{A}=$ skew $\boldsymbol{P} \in \mathfrak{s o}(3)$, the ansatz for the displacement field and the micro-rotation for the Cosserat model is

$$
\boldsymbol{u}\left(x_{2}\right)=\left(\begin{array}{c}
0 \\
u_{2}\left(x_{2}\right) \\
0
\end{array}\right), \quad \mathrm{D} \boldsymbol{u}\left(x_{2}\right)=\left(\begin{array}{ccc}
0 & 0 & 0 \\
0 & u_{2,2}\left(x_{2}\right) & 0 \\
0 & 0 & 0
\end{array}\right), \quad \boldsymbol{A}\left(x_{2}\right)=\left(\begin{array}{lll}
0 & 0 & 0 \\
0 & 0 & 0 \\
0 & 0 & 0
\end{array}\right)
$$

Since $\boldsymbol{A}=\mathbf{0}$, the Cosserat model is not able to catch any nonhomogeneous response for the uniaxial extension problem and classical solution (9) is retrieved.

The couple stress model $[10,11,16,19,23]$, which appears by constraining $\boldsymbol{A}=\operatorname{skew} \mathrm{D} \boldsymbol{u} \in \mathfrak{s o}(3)$ in the Cosserat model, is also not able to catch a nonhomogeneous response for the uniaxial extension problem since, due to the ansatz, we would have skew $\mathrm{D} \boldsymbol{u}=\mathbf{0}$ as it can be seen in Eq. (40).

\section{Uniaxial extension problem for the isotropic micro-void model in dislocation tensor format}

The strain energy for the isotropic micro-void continuum in dislocation tensor format can be obtained from the relaxed micromorphic model by formally letting $\mu_{\text {micro }} \rightarrow \infty$ (while keeping $\kappa_{\text {micro }}$ finite) and can be written as $[4,28]$ 
$W(\mathrm{D} \boldsymbol{u}, \omega, \operatorname{Curl}(\omega \mathbb{1}))=\mu_{\text {macro }}\|\operatorname{dev} \operatorname{sym} \mathrm{D} \boldsymbol{u}\|^{2}+\frac{\kappa_{\mathrm{e}}}{2} \operatorname{tr}^{2}(\mathrm{D} \boldsymbol{u}-\omega \mathbb{1})+\frac{\kappa_{\mathrm{micro}}}{2} \operatorname{tr}^{2}(\omega \mathbb{1})$

$$
+\frac{\mu L_{\mathrm{c}}^{2}}{2} a_{2}\|\operatorname{Curl}(\omega \mathbb{1})\|^{2} \text {. }
$$

Here, $\omega: \mathbb{R}^{3} \rightarrow \mathbb{R}$ is the additional scalar micro-void degree of freedom [4]. The equilibrium equations, in the absence of body forces, are

$$
\begin{aligned}
\operatorname{Div} \overbrace{\left[2 \mu_{\text {macro }} \operatorname{dev} \operatorname{sym} \operatorname{D} \boldsymbol{u}+\kappa_{\mathrm{e}} \operatorname{tr}(\mathrm{D} \boldsymbol{u}-\omega \mathbb{1}) \mathbb{1}\right]}^{\tilde{\sigma}:=} & =\mathbf{0}, \\
\frac{1}{3} \operatorname{tr}\left[\widetilde{\sigma}-\kappa_{\text {micro }} \operatorname{tr}(\omega \mathbb{1}) \mathbb{1}-\mu L_{\mathrm{c}}^{2} a_{2} \operatorname{Curl} \operatorname{Curl}(\omega \mathbb{1})\right] & =0 .
\end{aligned}
$$

and the positive definiteness conditions are

$$
\mu_{\text {macro }}>0, \quad \kappa_{\mathrm{e}}>0, \quad \kappa_{\text {micro }}>0, \quad \mu>0, \quad L_{\mathrm{c}}>0, \quad a_{2}>0 .
$$

According to the reference system shown in Fig. 1, the ansatz for the displacement field and the function $\omega\left(x_{2}\right)$ have to be

$$
\begin{aligned}
\boldsymbol{u}\left(x_{1}, x_{2}\right) & =\left(\begin{array}{c}
-x_{2} x_{3} \\
x_{1} x_{3} \\
0
\end{array}\right), \quad \omega\left(x_{2}\right) \mathbb{1}=\left(\begin{array}{ccc}
\omega\left(x_{2}\right) & 0 & 0 \\
0 & \omega\left(x_{2}\right) & 0 \\
0 & 0 & \omega\left(x_{2}\right)
\end{array}\right), \\
\mathrm{D} \boldsymbol{u}\left(x_{2}\right) & =\left(\begin{array}{ccc}
0 & 0 & 0 \\
0 & u_{2,2}\left(x_{2}\right) & 0 \\
0 & 0 & 0
\end{array}\right) .
\end{aligned}
$$

The boundary conditions for the uniaxial extension are

$$
u_{2}\left(x_{2}= \pm h / 2\right)= \pm \frac{\gamma h}{2}, \quad \omega\left(x_{2}= \pm h / 2\right)=0 .
$$

After substituting ansatz (44) into equilibrium equations (42) we obtain the following two differential equations

$$
\begin{array}{r}
\frac{1}{3}\left(3 \kappa_{\mathrm{e}}+4 \mu_{\text {macro }}\right) u_{2}^{\prime \prime}\left(x_{2}\right)-\kappa_{\mathrm{e}} \omega^{\prime}\left(x_{2}\right)=0, \\
\frac{2}{3} a_{2} \mu L_{\mathrm{c}}^{2} \omega^{\prime \prime}\left(x_{2}\right)+3 \kappa_{\mathrm{e}} u_{2}^{\prime}\left(x_{2}\right)-3\left(\kappa_{\mathrm{e}}+\kappa_{\text {micro }}\right) \omega\left(x_{2}\right)=0 .
\end{array}
$$

After applying boundary conditions Eq. (45), the solution in terms of $u_{2}\left(x_{2}\right)$ and $\omega\left(x_{2}\right)$ of system Eq. (46) is

$$
\begin{gathered}
u_{2}\left(x_{2}\right)=\frac{\frac{x_{2}}{h}-\frac{z_{1}}{f_{1}} \operatorname{sech}\left(\frac{f_{1} h}{2 L_{\mathrm{c}}}\right) \sinh \left(\frac{f_{1} x_{2}}{L_{\mathrm{c}}}\right) \frac{L_{\mathrm{c}}}{h}}{1-\frac{2 z_{1}}{f_{1}} \tanh \left(\frac{f_{1} h}{2 L_{\mathrm{c}}}\right) \frac{L_{\mathrm{c}}}{h}} h, \quad \omega\left(x_{2}\right)=\frac{z_{2}\left(1-\operatorname{sech}\left(\frac{f_{1} h}{2 L_{\mathrm{c}}}\right) \cosh \left(\frac{f_{1} x_{2}}{L_{\mathrm{c}}}\right)\right)}{1-\frac{2 z_{1}}{f_{1}} \tanh \left(\frac{f_{1} h}{2 L_{\mathrm{c}}}\right) \frac{L_{\mathrm{c}}}{h}} \boldsymbol{\gamma}, \\
f_{1}:=\sqrt{\frac{4 \mu_{\text {macro }}\left(\kappa_{\mathrm{e}}+\kappa_{\text {micro }}\right)+3 \kappa_{\mathrm{e}} \kappa_{\text {micro }}}{2 \mu a_{2}\left(3 \kappa_{\mathrm{e}}+4 \mu_{\text {macro }}\right)}}, \quad z_{1}:=\frac{3 \kappa_{\mathrm{e}}^{2}}{\left(\kappa_{\mathrm{e}}+\kappa_{\text {micro }}\right)\left(3 \kappa_{\mathrm{e}}+4 \mu_{\text {macro }}\right)}, \quad z_{2}:=\frac{\kappa_{\mathrm{e}}}{3\left(\kappa_{\mathrm{e}}+\kappa_{\text {micro }}\right)} .
\end{gathered}
$$

where $f_{1}>0, z_{1}>0$, and $z_{2}>0$ are strictly positive in order to match positive definiteness conditions Eq. (43), and the same reasoning applied in the relaxed micromorphic model sections still holds. The strain energy associated with this solution is

$$
\begin{aligned}
W(\boldsymbol{\gamma}) & =\int_{-h / 2}^{h / 2} W(\mathrm{D} \boldsymbol{u}, \boldsymbol{P}, \operatorname{Curl} \boldsymbol{P}) \\
& =\frac{1}{2}\left[\frac{\mu a_{2} f_{1}^{2} z_{2}^{2}\left(\frac{1}{f_{1}} \sinh \left(\frac{f_{1} h}{L_{\mathrm{c}}}\right) \frac{L_{\mathrm{c}}}{h}-1\right)}{\left(1-\frac{2 z_{1} 1}{f_{1}} \tanh \left(\frac{f_{1} h}{2 L_{\mathrm{c}}}\right) \frac{L_{\mathrm{c}}}{h}\right)^{2} \cosh ^{2}\left(\frac{f_{1} h}{2 L_{\mathrm{c}}}\right)}+\frac{\left(1+z 1+\cosh \left(\frac{f_{1} h}{L_{\mathrm{c}}}\right)-3 \frac{z_{1}}{f_{1}} \sinh \left(\frac{f_{1} h}{L_{\mathrm{c}}}\right) \frac{L_{\mathrm{c}}}{h}\right)}{2\left(1-\frac{2 z_{1} 1}{f_{1}} \tanh \left(\frac{f_{1} h}{2 L_{\mathrm{c}}}\right) \frac{L_{\mathrm{c}}}{h}\right)^{2} \cosh ^{2}\left(\frac{f_{\mathrm{f}} h}{2 L_{\mathrm{c}}}\right)}\left(\frac{\kappa_{\mathrm{e}} \kappa_{\text {micro }}}{\kappa_{\mathrm{e}}+\kappa_{\text {micro }}}+\frac{4 \mu_{\text {macro }}}{3}\right)\right] h \boldsymbol{\gamma}^{2} \\
& =\frac{1}{2} M_{\mathrm{w}} h \boldsymbol{\gamma}^{2} .
\end{aligned}
$$




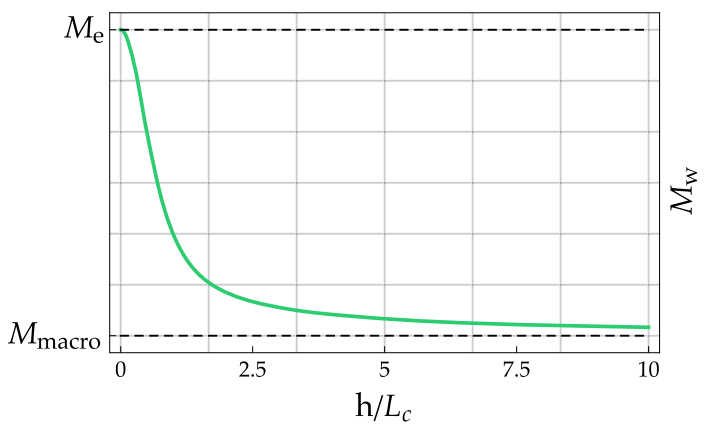

Fig. 3 Micro-void model. Extensional stiffness $M_{\mathrm{w}}$ while varying $L_{\mathrm{c}}$. The stiffness is bounded as $L_{\mathrm{c}} \rightarrow \infty(h \rightarrow 0)$ by $M_{\mathrm{e}}$. The values of the parameters used are: $\mu=1, \lambda_{\mathrm{e}}=1, M_{\mathrm{e}}=2, \kappa_{\text {micro }}=3, a_{2}=1 / 5$

The plot of the extensional stiffness $M_{\mathrm{w}}$ while varying $L_{\mathrm{c}}$ is shown in Fig. 3 .

The values of the extensional stiffness $M_{\mathrm{w}}$ for $L_{\mathrm{c}} \rightarrow 0$ and $L_{\mathrm{c}} \rightarrow \infty$ are

$$
\begin{aligned}
& \lim _{L_{\mathrm{c}} \rightarrow 0} M_{\mathrm{w}}=\frac{\kappa_{\mathrm{e}} \kappa_{\text {micro }}}{\kappa_{\mathrm{e}}+\kappa_{\text {micro }}}+\frac{4}{3} \mu_{\text {macro }}=\kappa_{\text {macro }}+\frac{4}{3} \mu_{\text {macro }}=2 \mu_{\text {macro }}+\lambda_{\text {macro }}=M_{\text {macro }}, \\
& \lim _{L_{\mathrm{c}} \rightarrow \infty} M_{\mathrm{w}}=\kappa_{\mathrm{e}}+\frac{4}{3} \mu_{\text {macro }}=\kappa_{\mathrm{e}}+\frac{4}{3} \mu_{\mathrm{e}}=2 \mu_{\mathrm{e}}+\lambda_{\mathrm{e}}=M_{\mathrm{e}},
\end{aligned}
$$

where $\mu_{\text {macro }}=\mu_{\mathrm{e}}$ for $\mu_{\text {micro }} \rightarrow \infty$, according to Eq. (29). We note that the extensional stiffness remains bounded as $L_{\mathrm{c}} \rightarrow \infty(h \rightarrow 0)$.

\section{Uniaxial extension problem for the classical isotropic micromorphic continuum without mixed terms}

The expression of the strain energy for the classical isotropic micromorphic continuum [7,17] without mixed terms (like $\langle\operatorname{sym} \boldsymbol{P}, \operatorname{sym}(\mathrm{D} \boldsymbol{u}-\boldsymbol{P})\rangle$, etc.) and simplified curvature expression $[25,27]$ can be written as:

$$
\begin{aligned}
W(\mathrm{D} \boldsymbol{u}, \boldsymbol{P}, \mathrm{D} \boldsymbol{P})= & \mu_{\mathrm{e}}\|\operatorname{sym}(\mathrm{D} \boldsymbol{u}-\boldsymbol{P})\|^{2}+\frac{\lambda_{\mathrm{e}}}{2} \operatorname{tr}^{2}(\mathrm{D} \boldsymbol{u}-\boldsymbol{P})+\mu_{\mathrm{c}} \| \text { skew }(\mathrm{D} \boldsymbol{u}-\boldsymbol{P}) \|^{2} \\
& +\mu_{\text {micro }}\|\operatorname{sym} \boldsymbol{P}\|^{2}+\frac{\lambda_{\text {micro }}}{2} \operatorname{tr}^{2}(\boldsymbol{P}) \\
& \left.+\frac{\mu L_{\mathrm{c}}^{2}}{2}\left(\tilde{a}_{1}\|\mathrm{D}(\operatorname{dev} \operatorname{sym} \boldsymbol{P})\|^{2}+\widetilde{a}_{2} \| \mathrm{D}(\text { skew } \boldsymbol{P})\left\|^{2}+\frac{2}{9} \widetilde{a}_{3}\right\| \mathrm{D}(\operatorname{tr}(\boldsymbol{P}) \mathbb{1}) \|^{2}\right)\right)
\end{aligned}
$$

while the equilibrium equations without body forces are the following:

$$
\begin{aligned}
& \operatorname{Div} \overbrace{\left[2 \mu_{\mathrm{e}} \operatorname{sym}(\mathrm{D} \boldsymbol{u}-\boldsymbol{P})+\lambda_{\mathrm{e}} \operatorname{tr}(\mathrm{D} \boldsymbol{u}-\boldsymbol{P}) \mathbb{1}+2 \mu_{\mathrm{c}} \operatorname{skew}(\mathrm{D} \boldsymbol{u}-\boldsymbol{P})\right]}^{\tilde{\boldsymbol{\sigma}}}=\mathbf{0}, \\
& \widetilde{\sigma}-2 \mu_{\text {micro }} \operatorname{sym} \boldsymbol{P}-\lambda_{\text {micro } \operatorname{tr}(\boldsymbol{P}) \mathbb{1}} \\
& \quad+\mu L_{\mathrm{c}}^{2} \operatorname{Div}\left[\tilde{a}_{1} \mathrm{D}(\operatorname{dev} \operatorname{sym} \boldsymbol{P})+\tilde{a}_{2} \mathrm{D}(\text { skew } \boldsymbol{P})+\frac{2}{9} \tilde{a}_{3} \mathrm{D}(\operatorname{tr}(\boldsymbol{P}) \mathbb{1})\right]=\mathbf{0},
\end{aligned}
$$

where $\left(\mu_{\mathrm{e}}, \kappa_{\mathrm{e}}=\lambda_{\mathrm{e}}+2 / 3 \mu_{\mathrm{e}}\right),\left(\mu_{\text {micro }}, \kappa_{\text {micro }}=\lambda_{\text {micro }}+2 / 3 \mu_{\text {micro }}\right), \mu_{\mathrm{c}}, L_{\mathrm{c}}>0$, and $\left(\widetilde{a}_{1}, \widetilde{a}_{2}, \widetilde{a}_{3}\right)>0$ in order to guarantee the positive definiteness of the energy. According to the reference system shown in Fig. 1, the ansatz for the displacement field and the classical micromorphic model is

$$
\begin{aligned}
\boldsymbol{u}\left(x_{2}\right) & =\left(\begin{array}{c}
0 \\
u_{2}\left(x_{2}\right) \\
0
\end{array}\right), \\
\mathrm{D} \boldsymbol{u}\left(x_{2}\right) & =\left(\begin{array}{ccc}
0 & 0 & 0 \\
0 & u_{2,2}\left(x_{2}\right) & 0 \\
0 & 0 & 0
\end{array}\right) .
\end{aligned}
$$


The boundary conditions for the uniaxial extension are assumed to be

$$
u_{2}\left(x_{2}= \pm h / 2\right)= \pm \frac{\boldsymbol{\gamma} h}{2}, \quad \boldsymbol{P}\left(x_{2}= \pm h / 2\right)=0 .
$$

The calculations are deferred to micro-strain model Sect. 8 since the ansatz, the equilibrium equations, and the boundary conditions are the same; therefore, the solution will also be the same.

\section{Uniaxial extension problem for the micro-strain model without mixed terms}

The micro-strain model $[9,12,31]$ is the classical Mindlin-Eringen [7,17] model particular case in which it is assumed a priori that the micro-distortion remains symmetric, $\boldsymbol{P}=\boldsymbol{S} \in \operatorname{Sym}(3)$.

The strain energy which we consider is $[25,27]$

$$
\begin{aligned}
W(\mathrm{D} \boldsymbol{u}, \boldsymbol{S}, \mathrm{D} \boldsymbol{S})= & \mu_{\mathrm{e}}\|(\operatorname{sym} \mathrm{D} \boldsymbol{u}-\boldsymbol{S})\|^{2}+\frac{\lambda_{\mathrm{e}}}{2} \operatorname{tr}^{2}(\mathrm{D} \boldsymbol{u}-\boldsymbol{S})+\mu_{\text {micro }}\|\boldsymbol{S}\|^{2}+\frac{\lambda_{\text {micro }}}{2} \operatorname{tr}^{2}(\boldsymbol{S}) \\
& +\frac{\mu L_{\mathrm{c}}^{2}}{2}\left(\widetilde{a}_{1}\|\mathrm{D}(\operatorname{dev} \boldsymbol{S})\|^{2}+\frac{2}{9} \widetilde{a}_{3}\|\mathrm{D}(\operatorname{tr}(\boldsymbol{S}) \mathbb{1})\|^{2}\right) .
\end{aligned}
$$

The chosen 2-parameter curvature expression represents a simplified isotropic curvature (the full isotropic curvature for the micro-strain model would still count 8 parameters [2]).

The equilibrium equations, in the absence of body forces, are therefore the following

$$
\begin{aligned}
& \operatorname{Div} \overbrace{\left[2 \mu_{\mathrm{e}}(\operatorname{sym} \mathrm{D} \boldsymbol{u}-\boldsymbol{S})+\lambda_{\mathrm{e}} \operatorname{tr}(\mathrm{D} \boldsymbol{u}-\boldsymbol{S}) \mathbb{1}\right]}^{\tilde{\sigma}:=}=\mathbf{0}, \\
& 2 \mu_{\mathrm{e}}(\operatorname{sym} \mathrm{D} \boldsymbol{u}-\boldsymbol{S})+\lambda_{\mathrm{e}} \operatorname{tr}(\mathrm{D} \boldsymbol{u}-\boldsymbol{S}) \mathbb{1}-2 \mu_{\text {micro }} \boldsymbol{S}-\lambda_{\text {micro }} \operatorname{tr}(\boldsymbol{S}) \mathbb{1} \\
& \quad+\mu L_{\mathrm{c}}^{2} \operatorname{sym} \operatorname{Div}\left[\widetilde{a}_{1} \mathrm{D}(\operatorname{dev} \boldsymbol{S})+\frac{2}{9} \widetilde{a}_{3} \mathrm{D}(\operatorname{tr}(\boldsymbol{S}) \mathbb{1})\right]=\mathbf{0}
\end{aligned}
$$

where $\left(\mu_{\mathrm{e}}, \kappa_{\mathrm{e}}=\lambda_{\mathrm{e}}+2 / 3 \mu_{\mathrm{e}}\right),\left(\mu_{\text {micro }}, \kappa_{\text {micro }}=\lambda_{\text {micro }}+2 / 3 \mu_{\text {micro }}\right), L_{\mathrm{c}}>0$, and $\left(\widetilde{a}_{1}, \widetilde{a}_{3}\right)>0$ in order to guarantee the positive definiteness of the energy. The boundary conditions for the uniaxial extension are assumed to be

$$
u_{2}\left(x_{2}= \pm h / 2\right)= \pm \frac{\boldsymbol{\gamma} h}{2}, \quad S\left(x_{2}= \pm h / 2\right)=0 .
$$

According to the reference system shown in Fig. 1, the ansatz for the displacement field and the microdistortion is (which coincides with classical micromorphic model Eq. (52))

$$
\begin{aligned}
\boldsymbol{u}\left(x_{2}\right) & =\left(\begin{array}{c}
0 \\
u_{2}\left(x_{2}\right) \\
0
\end{array}\right), \\
\mathrm{D} \boldsymbol{u}\left(x_{2}\right) & =\left(\begin{array}{ccc}
0 & 0 & 0 \\
0 & u_{2,2}\left(x_{2}\right) & 0 \\
0 & 0 & 0
\end{array}\right) .
\end{aligned}
$$

After substituting ansatz (57) into equilibrium equations (55) we obtain the following four differential equations

$$
\begin{aligned}
& M_{\mathrm{e}}\left(u_{2}^{\prime \prime}\left(x_{2}\right)-P_{22}^{\prime}\left(x_{2}\right)\right)-\lambda_{\mathrm{e}}\left(P_{11}^{\prime}\left(x_{2}\right)+P_{33}^{\prime}\left(x_{2}\right)\right)=0, \\
& \quad-\frac{2}{9} \mu L_{\mathrm{c}}^{2}\left(3 \widetilde{a}_{1}+\widetilde{a}_{3}\right) P_{11}^{\prime \prime}\left(x_{2}\right)+\frac{1}{9} \mu L_{\mathrm{c}}^{2}\left(3 \widetilde{a}_{1}-2 \widetilde{a}_{3}\right)\left(P_{22}^{\prime \prime}\left(x_{2}\right)+P_{33}^{\prime \prime}\left(x_{2}\right)\right) \\
& \quad+\left(M_{\mathrm{e}}+M_{\text {micro }}\right) P_{11}\left(x_{2}\right)+\left(\lambda_{\mathrm{e}}+\lambda_{\text {micro }}\right)\left(P_{22}\left(x_{2}\right)+P_{33}\left(x_{2}\right)\right)-\lambda_{\mathrm{e}} u_{2}^{\prime}\left(x_{2}\right)=0, \\
& \frac{1}{9} \mu L_{\mathrm{c}}^{2}\left(\left(3 \widetilde{a}_{1}-2 \widetilde{a}_{3}\right) P_{11}^{\prime \prime}\left(x_{2}\right)-2\left(3 \widetilde{a}_{1}+\widetilde{a}_{3}\right) P_{22}^{\prime \prime}\left(x_{2}\right)+\left(3 \widetilde{a}_{1}-2 \widetilde{a}_{3}\right) P_{33}^{\prime \prime}\left(x_{2}\right)\right) \\
& \quad+\left(M_{\mathrm{e}}+M_{\text {micro }}\right) P_{22}\left(x_{2}\right)-M_{\mathrm{e}} u_{2}^{\prime}\left(x_{2}\right)+\left(\lambda_{\mathrm{e}}+\lambda_{\text {micro }}\right)\left(P_{11}\left(x_{2}\right)+P_{33}\left(x_{2}\right)\right)=0,
\end{aligned}
$$



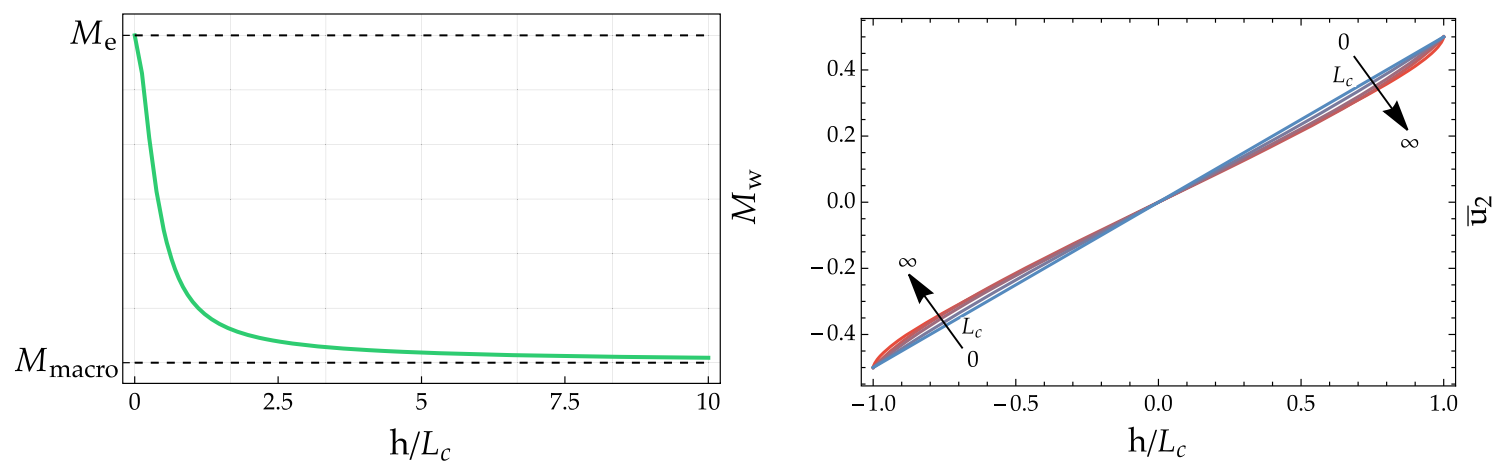

Fig. 4 Micro-strain model. (left) Extensional stiffness $M_{\mathrm{w}}$ while varying $L_{\mathrm{c}}$. The stiffness is bounded as $L_{\mathrm{c}} \rightarrow \infty(h \rightarrow 0)$ and converges to $M_{\mathrm{e}}$. The values of the parameters used are: $\mu=1, \lambda_{\text {macro }}=1, M_{\text {macro }}=3, \lambda_{\text {micro }}=9.69, M_{\text {micro }}=12$, $\tilde{a}_{1}=1 / 5, \widetilde{a}_{3}=1 / 6 ;$ (right) Displacement profile across the thickness of the dimensionless $\bar{u}_{2}=u_{2} /(\gamma h)$ for different values of $L_{\mathrm{c}}=\{0,3,5,10, \infty\}$. The values of the other parameters used in order to maximize the nonhomogeneous behavior are $\mu=1$, $\lambda_{\mathrm{e}}=11, M_{\mathrm{e}}=33, \lambda_{\text {micro }}=1.1, M_{\text {micro }}=3.3, \widetilde{a}_{1}=1, \widetilde{a}_{3}=1 / 6$

$$
\begin{aligned}
& \frac{1}{9} \mu L_{\mathrm{c}}^{2}\left(\left(3 \widetilde{a}_{1}-2 \widetilde{a}_{3}\right)\left(P_{11}^{\prime \prime}\left(x_{2}\right)+P_{22}^{\prime \prime}\left(x_{2}\right)\right)-2\left(3 \widetilde{a}_{1}+\widetilde{a}_{3}\right) P_{33}^{\prime \prime}\left(x_{2}\right)\right) \\
& \quad+\left(M_{\mathrm{e}}+M_{\text {micro }}\right) P_{33}\left(x_{2}\right)+\left(\lambda_{\mathrm{e}}+\lambda_{\text {micro }}\right)\left(P_{11}\left(x_{2}\right)+P_{22}\left(x_{2}\right)\right)-\lambda_{\mathrm{e}} u_{2}^{\prime}\left(x_{2}\right) \quad=0 .
\end{aligned}
$$

Being careful of substituting the system of differential equation with one in which Eq. (58) 2 and Eq. (58) 4 are replaced with their sum and their difference, respectively, we have

$$
\begin{aligned}
& M_{\mathrm{e}}\left(u_{2}^{\prime \prime}\left(x_{2}\right)-P_{22}^{\prime}\left(x_{2}\right)\right)-\lambda_{\mathrm{e}} f_{p}^{\prime}\left(x_{2}\right)=0, \\
& \quad-\frac{1}{9} \mu L_{\mathrm{c}}^{2}\left(\left(3 \widetilde{a}_{1}+4 \widetilde{a}_{3}\right) f_{p}^{\prime \prime}\left(x_{2}\right)+2\left(2 \widetilde{a}_{3}-3 \widetilde{a}_{1}\right) P_{22}^{\prime \prime}\left(x_{2}\right)\right) \\
& \quad+f_{p}\left(x_{2}\right)\left(M_{\mathrm{e}}+\lambda_{\mathrm{e}}+\lambda_{\text {micro }}+M_{\text {micro }}\right)+2\left(\lambda_{\mathrm{e}}+\lambda_{\text {micro }}\right) P_{22}\left(x_{2}\right)-2 \lambda_{\mathrm{e}} u_{2}^{\prime}\left(x_{2}\right)=0, \\
& \frac{1}{9} \mu L_{\mathrm{c}}^{2}\left(\left(3 \widetilde{a}_{1}-2 \widetilde{a}_{3}\right) f_{p}^{\prime \prime}\left(x_{2}\right)-2\left(3 \widetilde{a}_{1}+\widetilde{a}_{3}\right) P_{22}^{\prime \prime}\left(x_{2}\right)\right) \\
& \quad+\left(M_{\mathrm{e}}+M_{\text {micro }}\right) P_{22}\left(x_{2}\right)-M_{\mathrm{e}} u_{2}^{\prime}\left(x_{2}\right)+f_{p}\left(x_{2}\right)\left(\lambda_{\mathrm{e}}+\lambda_{\text {micro }}\right)=0, \\
& f_{m}\left(x_{2}\right)\left(M_{\mathrm{e}}-\lambda_{\mathrm{e}}-\lambda_{\text {micro }}+M_{\text {micro }}\right)-\widetilde{a}_{1} L_{\mathrm{c}}^{2} f_{m}^{\prime \prime}\left(x_{2}\right)=0,
\end{aligned}
$$

where $f_{p}\left(x_{2}\right):=P_{11}\left(x_{2}\right)+P_{33}\left(x_{2}\right)$ and $f_{m}\left(x_{2}\right):=P_{11}\left(x_{2}\right)-P_{33}\left(x_{2}\right)$. It is highlighted that Eq. (59) 4 is a homogeneous second-order differential equation depending only on $f_{m}\left(x_{2}\right)$ with homogeneous boundary conditions Eq. (56).

Also here, the fact that Eq. (59) $)_{4}$ is an independent equation has its meaning in the symmetry constraint of the uniaxial extensional problem in the direction along the $x_{2}$ - and $x_{3}$-axis, which requires that $P_{11}\left(x_{2}\right)=P_{33}\left(x_{2}\right)$.

The solution and the measure of the apparent stiffness are too complicated to be reported here, but nevertheless, it is possible to plot how the apparent stiffness behaves while changing $L_{\mathrm{c}}$ (see Fig. 4).

We note that the extensional stiffness remains bounded as $L_{\mathrm{c}} \rightarrow \infty(h \rightarrow 0)$ and converges to $M_{\mathrm{e}}$. The solution obtained for the micro-strain model for the uniaxial extension problem also holds for the classical micromorphic problem presented in Sect. 7.

\section{Uniaxial extension problem for the second gradient continuum}

The strain energy density for the isotropic second gradient with simplified curvature $[1,6,17,25,27]$ is

$$
\begin{aligned}
& W\left(\mathrm{D} \boldsymbol{u}, \mathrm{D}^{2} \boldsymbol{u}\right)=\mu_{\text {macro }}\|\operatorname{sym} \mathrm{D} \boldsymbol{u}\|^{2}+\frac{\lambda_{\text {macro }}}{2} \operatorname{tr}^{2}(\mathrm{D} \boldsymbol{u}) \\
& +\frac{\mu L_{\mathrm{c}}^{2}}{2}\left(\widetilde{a}_{1}\|\mathrm{D}(\operatorname{dev} \operatorname{sym} \mathrm{D} \boldsymbol{u})\|^{2}+\widetilde{a}_{2} \| \mathrm{D}(\text { skew D } \boldsymbol{u})\left\|^{2}+\frac{2}{9} \widetilde{a}_{3}\right\| \mathrm{D}(\operatorname{tr}(\mathrm{D} \boldsymbol{u}) \mathbb{1}) \|^{2}\right),
\end{aligned}
$$



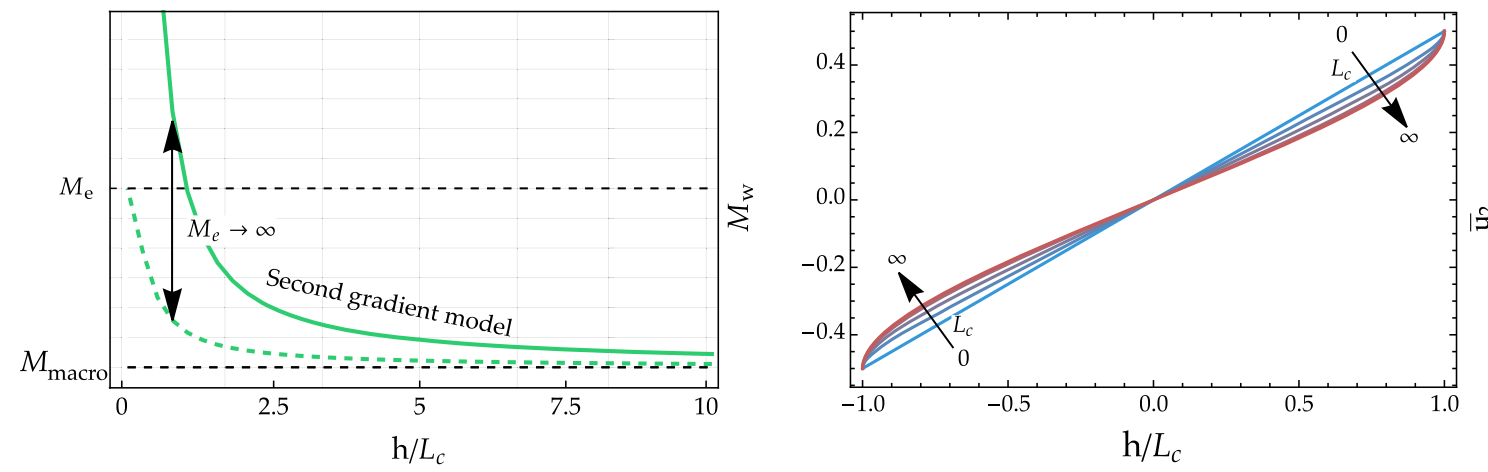

Fig. 5 Second gradient model. (left) Extensional stiffness $M_{\mathrm{w}}$ while varying $L_{\mathrm{c}}$. For the second gradient model (solid curve) the stiffness is unbounded as $L_{\mathrm{c}} \rightarrow \infty(h \rightarrow 0)$, while for the micro-strain model (dashed curve) the stiffness is bounded. The second gradient model can be obtained from the micro-strain model by formally letting $\mu_{\mathrm{e}}, \lambda_{\mathrm{e}} \rightarrow \infty$. The values of the parameters used are: $\mu=1, \mu_{\text {macro }}=1, \lambda_{\text {macro }}=2, \widetilde{a}_{3}=4 ;($ right $)$ Displacement profile across the thickness of the dimensionless $\bar{u}_{2}=u_{2} /(\gamma h)$ for different values of $L_{\mathrm{c}}=\{0,0.1,0.2,0.35, \infty\}$. The values of the other parameters used in order to maximize the nonhomogeneous behavior are $\mu=1, \lambda_{\text {micro }}=1, M_{\text {micro }}=1, \widetilde{a}_{3}=2$

while the equilibrium equations without body forces are the following:

$$
\begin{aligned}
& \operatorname{Div}\left[2 \mu_{\text {macro }} \operatorname{sym} \mathrm{D} \boldsymbol{u}+\lambda_{\text {macro }} \operatorname{tr}(\mathrm{D} \boldsymbol{u}) \mathbb{1}\right. \\
& \left.\quad-\mu L_{\mathrm{c}}^{2}\left(\widetilde{a}_{1} \operatorname{dev} \operatorname{sym} \boldsymbol{\Delta}(\mathrm{D} \boldsymbol{u})+\widetilde{a}_{2} \operatorname{skew} \boldsymbol{\Delta}(\mathrm{D} \boldsymbol{u})+\frac{2}{9} \widetilde{a}_{3} \operatorname{tr}(\boldsymbol{\Delta}(\mathrm{D} \boldsymbol{u})) \mathbb{1}\right)\right]=\mathbf{0},
\end{aligned}
$$

where $\left(\mu_{\text {macro }}, \kappa_{\text {macro }}, \mu, \widetilde{a}_{1}, \widetilde{a}_{3}\right)>0$ in order to guarantee the positive definiteness of the energy. Due to the uniaxial extension problem symmetry the following structure of $\boldsymbol{u}=\left(0, u_{2}\left(x_{2}\right), 0\right)^{T}$ has been chosen, which results in having only the component $u_{2,2}$ different from zero in the gradient of the displacement $\mathrm{D} \boldsymbol{u}$. The boundary conditions for the uniaxial extension are (see Fig. 1) assumed to be

$$
u_{2}\left(x_{2}= \pm h / 2\right)= \pm \frac{\gamma h}{2}, \quad u_{2}^{\prime}\left(x_{2}= \pm h / 2\right)=0
$$

After substituting the expression of the displacement field in Eq. (61), the nontrivial equilibrium equation reduces to

$$
\left(\lambda_{\text {micro }}+2 \mu_{\text {micro }}\right) u_{2}^{\prime \prime}\left(x_{2}\right)-\frac{1}{3} \widetilde{a}_{3} \mu L_{\mathrm{c}}^{2} u_{2}^{(4)}\left(x_{2}\right)=0 .
$$

After applying the boundary conditions to the solution of Eq. (63), it results that $u_{2}\left(x_{2}\right)$ is given by $[24,26]$

$$
u_{2}\left(x_{2}\right)=\frac{\frac{2 x_{2}}{h}-\frac{2}{f_{1}} \sinh \left(f_{1} \frac{x_{2}}{L_{\mathrm{c}}}\right) \operatorname{sech}\left(\frac{f_{1}}{2} \frac{h}{L_{\mathrm{c}}}\right) \frac{L_{\mathrm{c}}}{h}}{1-\frac{2}{f_{1}} \tanh \left(\frac{f_{1}}{2} \frac{h}{L_{\mathrm{c}}}\right) \frac{L_{\mathrm{c}}}{h}}, \quad f_{1}:=\sqrt{\frac{\lambda_{\text {macro }}+2 \mu_{\text {macro }}}{\mu \widetilde{a}_{3} / 3}} .
$$

where $f_{1}>0$ is strictly positive in order to match the positive definiteness conditions and the same reasoning applied in the relaxed micromorphic model sections still holds. Strain energy (61) becomes then

$$
W(\boldsymbol{\gamma})=\int_{0}^{h} W\left(\mathrm{D} \boldsymbol{u}, \mathrm{D}^{2} \boldsymbol{u}\right)=\frac{1}{2}\left[\frac{\overbrace{\lambda_{\text {macro }}+2 \mu_{\text {macro }}}^{M_{\text {macro }}}}{1-\frac{2}{f_{1}} \tanh \left(\frac{f_{1}}{2} \frac{h}{L_{\mathrm{c}}}\right) \frac{L_{\mathrm{c}}}{h}}\right] h \boldsymbol{\gamma}^{2}=\frac{1}{2} M_{\mathrm{w}} h \boldsymbol{\gamma}^{2} .
$$

The plot of the extensional stiffness $M_{\mathrm{w}}$ while varying $L_{\mathrm{c}}$ is shown in Fig. 5 . 


\title{
10 Conclusions
}

Only the second gradient formulation produces an unbounded apparent stiffness as $L_{\mathrm{c}} \rightarrow \infty(h \rightarrow 0)$, while for the other models different bounded limit stiffnesses are observed. For the second gradient model, because of its unboundedness stiffness, it can be more likely to have an instability in the parameters' fitting process on real structures: while being at a scale close to the singularity, a small changes in the geometrical or material properties of the sample may technically cause an arbitrarily large change in the values of the elastic coefficients. Therefore, the use of the second gradient model (or the classical micromorphic model in bending or torsional tests $[25,27]$ ) should be done with great care as regards the stable identification of parameters. These problems are avoided for the relaxed micromorphic model. The relaxed micromorphic model determines $\bar{M}=\frac{M_{\mathrm{e}} M_{\text {micro }}}{M_{\mathrm{e}}+M_{\text {micro }}}$, which is less than $M_{\text {micro }}$ and $M_{\mathrm{e}}$, while the micro-strain model determines $M_{\mathrm{e}}$ as limit stiffness. The Cosserat model is not able to catch a nonhomogeneous solution and provides no size effect. The different limit stiffnesses for the relaxed micromorphic model versus the full micromorphic and micro-strain model approach, respectively, suggest that the meaning of classical experimental tests does not have an unambiguous deformation and micro-deformation solution field anymore, and this is due to the fact that we can have different boundary conditions on the components of the micro-distortion tensor depending on what each model requires to constrain. This allows the existence of different uniaxial extension-like problems and not just one like for a classical Cauchy material.

\begin{abstract}
Acknowledgements Angela Madeo and Gianluca Rizzi acknowledge the support from the European Commission through the funding of the ERC Consolidator Grant META-LEGO, N ${ }^{\circ}$ 101001759. Angela Madeo and Gianluca Rizzi acknowledge funding from the French Research Agency ANR, "METASMART" (ANR-17CE08-0006). Hassam Khan acknowledges the support of the German Academic Exchange Service (DAAD) and the Higher Education Commission of Pakistan (HEC). Ionel Dumitrel Ghiba acknowledges the support from a grant of the Romanian Ministry of Research and Innovation, CNCS-UEFISCDI, project number PN-III-PN-III-P1-1.1-TE-2021-0783, within PNCDI III. Patrizio Neff acknowledges the support in the framework of the DFG-Priority Programme 2256 "Variational Methods for Predicting Complex Phenomena in Engineering Structures and Materials", Neff 902/10-1, Project No. 440935806.

Open Access This article is licensed under a Creative Commons Attribution 4.0 International License, which permits use, sharing, adaptation, distribution and reproduction in any medium or format, as long as you give appropriate credit to the original author(s) and the source, provide a link to the Creative Commons licence, and indicate if changes were made. The images or other third party material in this article are included in the article's Creative Commons licence, unless indicated otherwise in a credit line to the material. If material is not included in the article's Creative Commons licence and your intended use is not permitted by statutory regulation or exceeds the permitted use, you will need to obtain permission directly from the copyright holder. To view a copy of this licence, visit http://creativecommons.org/licenses/by/4.0/.
\end{abstract}

Funding Open Access funding enabled and organized by Projekt DEAL.

\section{A The limit $L_{\mathrm{c}} \rightarrow \infty$ for the relaxed micromorphic model}

The limit of the energy, Eq. (11), for $L_{\mathrm{c}} \rightarrow \infty$, requires that $\|\operatorname{Curl} \boldsymbol{P}\|=0$, which implies that $\boldsymbol{P}=\mathrm{D} \zeta$, for some $\zeta: \Omega \rightarrow \mathbb{R}^{3}$. Energy Eq. (11) now becomes

$$
W(\mathrm{D} \boldsymbol{u}, \mathrm{D} \zeta)=\mu_{\mathrm{e}}\|\operatorname{sym}(\mathrm{D} \boldsymbol{u}-\mathrm{D} \zeta)\|^{2}+\frac{\lambda_{\mathrm{e}}}{2} \operatorname{tr}^{2}(\mathrm{D} \boldsymbol{u}-\boldsymbol{P})+\mu_{\text {micro }}\|\operatorname{sym} \mathrm{D} \zeta\|^{2}+\frac{\lambda_{\text {micro }}}{2} \operatorname{tr}^{2}(\mathrm{D} \zeta),
$$

and that Eq. (13) turns into

$$
\operatorname{Div} \overbrace{\left[\begin{array}{c}
\left.2 \mu_{\mathrm{e}} \operatorname{sym}(\mathrm{D} \boldsymbol{u}-\mathrm{D} \zeta)+\lambda_{\mathrm{e}} \operatorname{tr}(\mathrm{D} \boldsymbol{u}-\mathrm{D} \zeta) \mathbb{1}\right] \\
\tilde{\sigma}-2 \mu_{\text {micro }} \operatorname{sym} \mathrm{D} \zeta-\lambda_{\text {micro }} \operatorname{tr}(\mathrm{D} \zeta) \mathbb{1}
\end{array}=\mathbf{0},\right.}^{\tilde{\boldsymbol{\sigma}}:=}=\mathbf{0},
$$

with consistent coupling boundary condition $\mathrm{D} \boldsymbol{u} \cdot \boldsymbol{\tau}=\mathrm{D} \boldsymbol{\zeta} \cdot \boldsymbol{\tau}$. Given Eq. (66) 1 , Eq. (66) $)_{2}$ reduces to be

$$
\operatorname{Div}\left[2 \mu_{\text {micro }} \operatorname{sym} \mathrm{D} \zeta+\lambda_{\text {micro }} \operatorname{tr}(\mathrm{D} \zeta) \mathbb{1}\right]=\mathbf{0},
$$

which, for the uniaxial extension problem with boundary condition $u_{2}\left(x_{2}= \pm h / 2\right)= \pm \boldsymbol{\gamma} h / 2$, is equivalent to

$$
\mathrm{D} \zeta=\left(\begin{array}{ccc}
0 & 0 & 0 \\
0 & a & 0 \\
0 & 0 & 0
\end{array}\right), \quad \mathrm{D} \boldsymbol{u}=\left(\begin{array}{ccc}
0 & \boldsymbol{\gamma} & 0 \\
0 & 0 & 0 \\
0 & 0 & 0
\end{array}\right)
$$


where $a$ is an arbitrary constant. This solution to Eq. (66) is therefore not unique. Inserting $\mathrm{D} \boldsymbol{u}$ and $\mathrm{D} \zeta$ from Eq. (68) in Eq. (65), the following energy expression is recovered

$$
I(a)=\frac{1}{2}\left(2 a^{2} M_{\text {micro }}+2 M_{\mathrm{e}}(a-\gamma)^{2}\right),
$$

which has to be minimized with respect to $a$ in order to remove the nonuniqueness of equilibrium system Eq. (66), which means that the following relation

$$
\frac{\partial}{\partial a}\left(a^{2} M_{\text {micro }}+M_{\mathrm{e}}(a-\gamma)^{2}\right)=2 a\left(M_{\mathrm{e}}+M_{\text {micro }}\right)-2 \gamma M_{\mathrm{e}}=0
$$

has to be satisfied. The solution of Eq. (70) is $a_{\mathrm{min}}=\frac{M_{\mathrm{e}}}{M_{\mathrm{e}}+M_{\text {micro }}} \gamma$. Finally it is possible to substitute $a_{\mathrm{min}}$ into Eq. (68) obtaining

$$
\mathrm{D} \zeta=\left(\begin{array}{ccc}
0 & 0 & 0 \\
0 & \frac{M_{\mathrm{e}}}{M_{\mathrm{e}}+M_{\text {micro }}} \boldsymbol{\gamma} & 0 \\
0 & 0 & 0
\end{array}\right), \quad \mathrm{D} \boldsymbol{u}=\left(\begin{array}{ccc}
0 & \boldsymbol{\gamma} & 0 \\
0 & 0 & 0 \\
0 & 0 & 0
\end{array}\right) .
$$

Solution Eq. (71) satisfies the equilibrium equations, the boundary conditions, and the minimum energy requirement. The expression of the energy now becomes

$$
W(\boldsymbol{\gamma})=\int_{-h / 2}^{h / 2} W(\mathrm{D} \boldsymbol{u}, \mathrm{D} \zeta)=\frac{1}{2} \frac{M_{\mathrm{e}} M_{\text {micro }}}{M_{\mathrm{e}}+M_{\text {micro }}} h \boldsymbol{\gamma}^{2}=\frac{1}{2} \bar{M} h \boldsymbol{\gamma}^{2},
$$

with $\bar{M}=\frac{M_{\mathrm{e}} M_{\text {micro }}}{M_{\mathrm{e}}+M_{\text {micro }}}$ the extensional stiffness for the relaxed micromorphic when $L_{\mathrm{c}} \rightarrow \infty$.

\section{References}

1. Altenbach, H., Müller, W.H., Abali, B.E.: Higher Gradient Materials and Related Generalized Continua. Springer, Berlin (2019)

2. Barbagallo, G., Madeo, A., d'Agostino, M., Abreu, R., Ghiba, I., Neff, P.: Transparent anisotropy for the relaxed micromorphic model: macroscopic consistency conditions and long wave length asymptotics. Int. J. Solids Struct. 120, 7-30 (2017)

3. Cosserat, E., Cosserat, F.: Théorie des corps déformables. A. Hermann et fils (reprint 2009), Paris (1909)

4. Cowin, S., Nunziato, J.: Linear elastic materials with voids. J. Elast. 13(2), 125-147 (1983)

5. De Cicco, S., Nappa, L.: Torsion and flexure of microstretch elastic circular cylinders. Int. J. Eng. Sci. 35(6), 573-583 (1997)

6. Dell'Isola, F., Sciarra, G., Vidoli, S.: Generalized Hooke's law for isotropic second gradient materials. Proc. R. Soc. A Math. Phys. Eng. Sci. 465(2107), 2177-2196 (2009)

7. Eringen, A.C.: Mechanics of micromorphic continua. Mechanics of generalized continua, pp. 18-35. Springer, Berlin (1968)

8. Fantuzzi, N., Leonetti, L., Trovalusci, P., Tornabene, F.: Some novel numerical applications of Cosserat continua. Int. J. Comput. Methods 15(06), 1850054 (2018)

9. Forest, S., Sievert, R.: Nonlinear microstrain theories. Int. J. Solids Struct. 43(24), 7224-7245 (2006)

10. Ghiba, I., Neff, P., Madeo, A., Münch, I.: A variant of the linear isotropic indeterminate couple-stress model with symmetric local force-stress, symmetric nonlocal force-stress, symmetric couple-stresses and orthogonal boundary conditions. Math. Mech. Solids 22(6), 1221-1266 (2017)

11. Hadjesfandiari, A., Dargush, G.: Comparison of theoretical elastic couple stress predictions with physical experiments for pure torsion (2016). arXiv:1605.02556

12. Hütter, G., Mühlich, U., Kuna, M.: Micromorphic homogenization of a porous medium: elastic behavior and quasibrittle damage. Contin. Mech. Thermodyn. 27(6), 1059-1072 (2015)

13. Izadi, R., Tuna, M., Trovalusci, P., Ghavanloo, E.: Torsional characteristics of carbon nanotubes: micropolar elasticity models and molecular dynamics simulation. Nanomaterials. 11(2), 453 (2021)

14. Jeong, J., Ramézani, H., Münch, I., Neff, P.: A numerical study for linear isotropic Cosserat elasticity with conformally invariant curvature. Z. Angew. Math. Mech. 89(7), 552-569 (2009)

15. Kirchner, N., Steinmann, P.: Mechanics of extended continua: modeling and simulation of elastic microstretch materials. Comput. Mech. 40(4), 651-666 (2007)

16. Koiter, W.: Couple stresses in the theory of elasticity: I and II. Proc. Kon. Ned. Akad. Wetensch. Ser. B 67, 17-44 (1964)

17. Mindlin, R.: Micro-structure in linear elasticity. Arch. Ration. Mech. Anal. 16(1), 51-78 (1964)

18. Neff, P.: The Cosserat couple modulus for continuous solids is zero viz the linearized Cauchy-stress tensor is symmetric. Z. Angew. Math. Mech. 86(11), 892-912 (2006)

19. Neff, P., Ghiba, I., Madeo, A., Münch, I.: Correct traction boundary conditions in the indeterminate couple stress model (2015). arXiv: 1504.00448 
20. Neff, P., Ghiba, I., Madeo, A., Placidi, L., Rosi, G.: A unifying perspective: the relaxed linear micromorphic continuum. Continu. Mech. Thermodyn. 26(5), 639-681 (2014)

21. Neff, P., Jeong, J.: A new paradigm: the linear isotropic Cosserat model with conformally invariant curvature energy. Z. Angew. Math. Mech. 89(2), 107-122 (2009)

22. Neff, P., Jeong, J., Münch, I., Ramezani, H.: Mean field modeling of isotropic random Cauchy elasticity versus microstretch elasticity. Z. Angew. Math. Phys. 60(3), 479-497 (2009)

23. Neff, P., Münch, I., Ghiba, I., Madeo, A.: On some fundamental misunderstandings in the indeterminate couple stress model. A comment on recent papers of AR Hadjesfandiari and GF Dargush. Int. J. Solids Struct. 81, 233-243 (2016)

24. Rizzi, G., Dal Corso, F., Veber, D., Bigoni, D.: Identification of second-gradient elastic materials from planar hexagonal lattices. Part II: mechanical characteristics and model validation. Int. J. Solids Struct. 176, 19-35 (2019)

25. Rizzi, G., Hütter, G., Madeo, A., Neff, P.: Analytical solutions of the cylindrical bending problem for the relaxed micromorphic continuum and other generalized continua. Contin. Mech. Thermodyn. 1-35 (2021). arXiv:2012.10391

26. Rizzi, G., Dal Corso, F., Veber, D., Bigoni, D.: Identification of second-gradient elastic materials from planar hexagonal lattices. Part I: analytical derivation of equivalent constitutive tensors. Int. J. Solids Struct. 176, 1-18 (2019)

27. Rizzi, G., Hütter, G., Khan, H., Ghiba, I. D., Madeo, A., Neff, P.: Analytical solution of the cylindrical torsion problem for the relaxed micromorphic continuum and other generalized continua (including full derivations). Math. Mech. Solids (2021) (to appear). arXiv:2104.11322

28. Rizzi, G., Hütter, G., Madeo, A., Neff, P.: Analytical solutions of the simple shear problem for micromorphic models and other generalized continua. Arch. Appl. Mech. 91(5), 2237-2254 (2021)

29. Rueger, Z., Lakes, R.: Strong Cosserat elasticity in a transversely isotropic polymer lattice. Phys. Rev. Lett. 120(6), 065501 (2018)

30. Scalia, A.: Extension, bending and torsion of anisotropic microstretch elastic cylinders. Math. Mech. Solids 5(1), 31-40 (2000)

31. Shaat, M.: A reduced micromorphic model for multiscale materials and its applications in wave propagation. Compos. Struct. 201, 446-454 (2018)

32. Shaat, M., Ghavanloo, E., Fazelzadeh, S.A.: Review on nonlocal continuum mechanics: physics, material applicability, and mathematics. Mech. Mater. 150, 103587 (2020)

33. Shekarchizadeh, N., Abali, B.E., Barchiesi, E., Bersani. A.M.: Inverse analysis of metamaterials and parameter determination by means of an automatized optimization problem. Zeitschrift für Angewandte Mathematik und Mechanik (2021)

34. Triawan, F., Kishimoto, K., Adachi, T., Inaba, K., Nakamura, T., Hashimura, T.: The elastic behavior of aluminum alloy foam under uniaxial loading and bending conditions. Acta Mater. 60(6-7), 3084-3093 (2012)

35. Yao, H., Yun, G., Bai, N., Li, J.: Surface elasticity effect on the size-dependent elastic property of nanowires. J. Appl. Phys. 111(8), 083506 (2012)

36. Zhao, H., Min, K., Aluru, N.R.: Size and chirality dependent elastic properties of graphene nanoribbons under uniaxial tension. Nano Lett. 9(8), 3012-3015 (2009)

Publisher's Note Springer Nature remains neutral with regard to jurisdictional claims in published maps and institutional affiliations. 